\title{
Tropical cyclones in a T159 resolution global climate model: comparison with observations and re-analyses
}

\author{
By L. BENGTSSON ${ }^{1,2}$, K. I. HODGES ${ }^{1 *}$ and M. ESCH ${ }^{2},{ }^{1}$ Environmental Systems Science Centre \\ (ESSC), University of Reading, Whiteknights, PO Box 238, Reading, UK; ${ }^{2}$ Max Planck Institute for Meteorology, \\ Hamburg, Germany
}

(Manuscript received 27 September 2006; in final form 29 January 2007)

\begin{abstract}
Tropical cyclones have been investigated in a T159 version of the MPI ECHAM5 climate model using a novel technique to diagnose the evolution of the three-dimensional vorticity structure of tropical cyclones, including their full life cycle from weak initial vortices to their possible extra-tropical transition. Results have been compared with re-analyses [the European Centre for Medium-Range Weather Forecasts (ECMWF) 40-yr Re-analysis (ERA40) and Japanese 25 yr re-analysis (JRA25)] and observed tropical storms during the period 1978-1999 for the Northern Hemisphere. There is no indication of any trend in the number or intensity of tropical storms during this period in ECHAM5 or in re-analyses but there are distinct inter-annual variations. The storms simulated by ECHAM5 are realistic both in space and time, but the model and even more so the re-analyses, underestimate the intensities of the most intense storms (in terms of their maximum wind speeds). There is an indication of a response to El Niño-Southern Oscillation (ENSO) with a smaller number of Atlantic storms during El Niño in agreement with previous studies.

The global divergence circulation responds to El Niño by setting up a large-scale convergence flow, with the centre over the central Pacific with enhanced subsidence over the tropical Atlantic. At the same time there is an increase in the vertical wind shear in the region of the tropical Atlantic where tropical storms normally develop. There is a good correspondence between the model and ERA40 except that the divergence circulation is somewhat stronger in the model. The model underestimates storms in the Atlantic but tends to overestimate them in the Western Pacific and in the North Indian Ocean.

It is suggested that the overestimation of storms in the Pacific by the model is related to an overly strong response to the tropical Pacific sea surface temperature (SST) anomalies. The overestimation in the North Indian Ocean is likely to be due to an over prediction in the intensity of monsoon depressions, which are then classified as intense tropical storms. Nevertheless, overall results are encouraging and will further contribute to increased confidence in simulating intense tropical storms with high-resolution climate models.
\end{abstract}

\section{Introduction}

In a recent paper by Bengtsson et al. (2006) it was shown that the Max Planck Institute (MPI) coupled climate model ECHAM5/MPI-OM was capable of realistically reproducing the geographical distribution of transient tropical and extra-tropical atmospheric eddies. The results compared well with those using the European Centre for Medium-Range Weather Forecasts (ECMWF) 40-yr Re-analysis (ERA40) data (Uppala et al., 2005). Results showed that there was a comparatively small change between the present climate and a future climate at the end of the present century, based on the Intergovernmental Panel

\footnotetext{
* Corresponding author.

e-mail: kih@mail.nerc-essc.ac.uk

DOI: $10.1111 / \mathrm{j} .1600-0870.2007 .00236 . x$
}

on Climate Change (IPCC) Special Report on Emission Scenarios (SRES) scenario A1B (Nakicenovic et al., 2000), with respect to the total number and intensities of vortices on a global scale. However, significant changes were found regionally, such as an indication of a pole-ward trend of the extra-tropical eddies and a marked sensitivity to El Niño-Southern Oscillation (ENSO) of the transient tropical eddies.

General circulation models (GCM) have convincingly demonstrated that they can generate distinct warm core eddies which can be interpreted as tropical storms or at least as onset vortices required to initiate an organized tropical storm or a hurricane, (Henderson-Sellers et al., 1998). Here we use the term tropical cyclone to refer to intense storms in any ocean basin. Similarly, global operational forecasting systems, such as at ECMWF, the United Kingdom Met Office, National Centers for Environmental Prediction (NCEP) and the Japan Meteorological Agency 
(JMA) are making steady improvements in predicting tropical cyclone trajectories while generally underestimating their intensity (e.g. DeMaria et al., 2005). The dominant reason for this appears to be insufficient horizontal resolution of present operational models (Bender and Ginis, 2000) but presumably also the handling of convective processes in the models (Shen et al., 2006a).

Because of the enormous social and economic impact of tropical cyclones, it is of the utmost importance to explore how credible the model generated storms are. If we can establish measures of credibility of model-generated storms in the present climate, it will provide important support for assessing the credibility of model generated storms in a future climate. The past approach to identifying tropical eddies in an extended model run over a period of many years has been to use specific selection criteria with respect to the intensity and structure of the storms as well as their lifetime. Such selection criteria typically include 850 hpa vorticity, surface pressure, maximum surface wind speed and a well defined warm core identified from the temperature anomalies at several levels through the troposphere. Most of the selection criteria used follow those suggested by Bengtsson et al. (1995). A summary of past selection criteria can be found in Chauvin et al. (2006). Studies based on these selection criteria generally only identify and track the storms when these selection criteria are satisfied, which results in the early and latter stages of the storms lifecycle being excluded, although the tracks can be extended forwards and backwards in time (Camargo and Zebiak, 2002). We will take a different approach here to capture the full storm lifecycle from a tropical onset vortex to possible extratropical transition.

Most previous studies have used comparatively coarse resolutions, typically T42 or equivalent [Broccoli and Manabe, 1990 (R30), Haarsma et al., 1993 (R30), Camargo and Zebiak, 2002 (T42) and references therein], although Bengtsson et al. (1995, 1996) and Sugi et al. (2002) have used data from model integrations at a higher spectral resolution of triangular truncation 106 (T106). More recently, there have been a few studies exploring the capability of global models, integrated at much higher resolutions, in predicting and simulating tropical cyclones. These include prediction studies, for example, Ohfuchi et al. (2004) who used a resolution of $10 \mathrm{~km}$ and Shen et al. (2006a, b) who used a resolution of $1 / 4$ and $1 / 8$ of a degree. It also includes climate simulation experiments, such as Chauvin et al. (2006), who used a spectral transform resolution of T319, and Oouchi et al. (2006) who used a resolution of $20 \mathrm{~km}$. The results of the high-resolution GCM experiments confirm the experience gained from high resolution limited area models (Bengtsson et al., 1997; Knutson and Tuleya, 2004) that given sufficient horizontal resolution, models are increasingly capable of reproducing the detailed structure of circulation and intensity typical of tropical cyclones.

The purpose of this study is to introduce a new approach for objectively identifying tropical cyclones in gridded data sets, based on a simpler set of identification criteria, and then to use this to study the performance of the atmosphere only ECHAM5 model in multidecadal integrations at horizontal resolutions of T159. This will act as a prequel to a study of the impact of climate change on tropical cyclones at a range of resolutions using the same model, to be reported in a second publication (Bengtsson et al., 2007). We will employ the notation Tropical Cyclones (TC), as opposed to the term Hurricane Type Vortices (HTV) as used in previous studies (Bengtsson et al., 1995, Chauvin et al., 2006), since storms are identified throughout the tropics. It is left to the context as to whether TC is singular or plural.

Here we investigate a T159 experiment using observed sea surface temperatures (SST) that are practically identical to those used in ERA 40. We compare the model generated storms with assimilated TC in re-analyses and with observed tropical cyclones obtained as the 'best track' data for each ocean basin produced by the international warning centres (see Klotzback, 2006). We restrict the study of the TC to the Northern Hemisphere (NH) but search for TC through the whole year. In order to identify TC we employ a new search method making use of the full three-dimensional (3-D) structure of the eddies. We track the eddies during their full life cycle, from their generation to their decay as extra-tropical eddies. As the time resolution of the model and re-analyses databases is $6 \mathrm{hr}$, and the same criteria are used to identify the TC in the model and re-analyses, this allows a detailed intercomparison to be made between the model and the recent re-analyses of ERA40 and the Japanese 25 yr re-analysis (JRA25) (Onogi et al., 2005). We also compare the model TC with the observed tropical cyclones, though because different criteria are used for identification of the observed tropical cyclones this comparison is more problematic. In particular, since we use the intensity and structure of the vorticity field to identify the TC it is a problem to compare the TC with actual tropical cyclones as identified by the Tropical Warning Centers (TWC) since vorticity is not an easily observed quantity. However, there is a close relation between the maximum wind speed and the maximum vorticity in both the re-analysis data and ECHAM5, which can be used as a basis for comparing with the observed TC. As well as the differences in identification between the method used here and operationally there are also differences in how particular quantities are measured. For example, wind speeds as a measure of intensity of the storms, are often measured differently by the different TWC, i.e. one minute sustained winds or 10 minute sustained surface winds, where as for our model data, we only have data every $6 \mathrm{hr}$ which may be seen to represent averaged conditions of the order of $30 \mathrm{~min}$ and typical for an area of some $100 \mathrm{~km}$. Hence, the comparison with the best tracks from the TWCs should be seen as tentative only and mainly qualitative.

The scientific objectives of the study are as follows:

(1) To identify what are the systematic differences between $\mathrm{TC}$ in the model experiment, in the re-analyses and the observed 
TC including their structure and behaviour and their distribution in time and space.

(2) To determine what are the likely causes of the differences between the model simulated TC and the TC in the re-analyses and observations.

(3) To explore whether the model can reproduce interannual variations of the TC and the likely causes for this variation.

\section{The model, experiments and data}

The model used to produce the data for this study is the same as that used in the study of Bengtsson et al. (2006) and described by Roeckner et al. (2003), where further details can be obtained. The sensitivity of this model to horizontal and vertical resolution has previously been explored by Roeckner et al. (2006). This is a spectral model and for the data used here it has been run in atmosphere only mode at spectral resolution of triangular truncation 159 with 31 levels in the vertical (T159L31), using the observed SST's for the period 1978-1999, based on the Atmospheric Model Intercomparison Project, version 2 (AMIP2) protocol (WGNE, 1996). The results from the AMIP2 experiment are contrasted with those from the recent re-analyses of ERA40 (Uppala et al., 2005) and JRA25 (Onogi et al., 2005) for the same period. Both ERA40 and JRA25 assimilate historical observations with a GCM using modern Numerical Weather Prediction systems. Both are based on spectral models and both use (3-D) data assimilation (3-D Var.). ERA40 is integrated at a horizontal spectral resolution of T159 with 61 levels in the vertical (T159L61), i.e. the same horizontal resolution as the AMIP2, ECHAM5 experiment, where as the JRA25 re-analysis is integrated at the lower resolution of T106 with 40 levels in the vertical (T106L40). Both use the direct assimilation of satellite radiances. Both the re-analyses and model data are available six hourly. The model experiment and re-analyses are also compared with the observed tropical storms over the period obtained as best track data produced by the Tropical Warning Centers (TWC) for each ocean basin and obtained from NCAR Data Support Section (http://dss.ucar.edu). Throughout the paper, the main comparisons will be made between ERA40, ECHAM5 and the observations with a short contrast between ERA40 and JRA25 being left till the end.

\section{Tracking methodology and TC identification}

The initial identification and tracking closely follows that used for the study of Thorncroft and Hodges (2001) and also used in other studies of easterly wave and tropical cyclone activity (Hodges et al., 2003, Bengtsson et al., 2006). This identifies tropical vortices in the $\mathrm{NH}$ as maxima in the $850 \mathrm{hPa}$ relative vorticity field, available every $6 \mathrm{hr}$, with values greater than $0.5 \times$ $10^{-5} \mathrm{~s}^{-1}$ at a spectral resolution of T42. Relative vorticity is used since it focuses on smaller spatial scales than other fields, such as pressure, and is hence a better indicator of tropical vortices. The reduced resolution is used to remove the very small subsyn- optic spatial scales which can result in multiple vorticity centres and centres associated with subsynoptic phenomena which can lead to tracking errors. To capture as much of the TC lifecycle as possible, the region explored covers the whole of the tropics from the equator and extended to the extra-tropics at $60^{\circ} \mathrm{N}$. At this stage no discrimination is made between TC and other synoptic systems, every system that satisfies the identification and tracking criteria are obtained. All vortices are retained that have lifetimes greater than $2 \mathrm{~d}$ for further analysis. There is no restriction on the displacement distance of the vortices. Although, this approach entails identifying and tracking a lot of systems, many of which are not TC it does ensure that we capture as much of the TC lifetimes as possible.

Since we want to use the vertical structure of the vortices to identify those with a warm core structure and to explore the impact of resolution on the properties of the TC, all the available tracks are referenced to other fields and levels using the following procedure. To identify the vortices with warm core structures, we reference the available tracks to the vorticity field at the 850 , $600,500,400,300$ and $250 \mathrm{hPa}$ levels at a resolution of T63. This is done in an iterative way by starting at $850 \mathrm{hPa}$ and using the T42 maxima as initial starting points for a steepest ascent maximization of the T63 $850 \mathrm{hPa}$ field interpolated using B-splines. The $850 \mathrm{hPa}$ maxima are then used as starting points for the maximization at $600 \mathrm{hPa}$ and so on up to $250 \mathrm{hPa}$. Additionally, the same procedure is used to obtain properties along the tracks, at the full resolution, of the $850 \mathrm{hPa}$ vorticity, $925 \mathrm{hPa}$ maximum wind speed and the minimum pressure (steepest descent). The $\max /$ minimization is performed within a $5^{\circ}$ geodesic radius for the vorticity and pressure and $6^{\circ}$ geodesic radius for the winds. Both the position and the intensity values of the new $\mathrm{max} / \mathrm{min}$ are stored. If a maxima or minima cannot be found, the value at the T42 centre is computed and stored and the positional values are tagged with a missing value. For the vertical structure, the T63 resolution is used since this is the lowest resolution data we have for our resolution studies, it also helps in providing a smoother picture of the vertical structure than at the full resolution, although in principle, the full resolution could be used. For resolution studies it helps in the comparison to perform the identification at a common resolution.

The criteria used to identify the TC are as follows:-

(1) Lifetime $\geq 2 \mathrm{~d}$ (8 time steps).

(2) Cyclogenesis, defined by first identification, must occur in $(0,20) \mathrm{N}$ over land and $(0-30) \mathrm{N}$ over oceans.

(3) The maximum T63 intensity of relative vorticity at 850 $\mathrm{hPa}$ during the lifetime $\geq \xi_{\text {I }}$ for some chosen value of $\xi_{\mathrm{I}}$.

(4) There must be a T63 vorticity maxima at each level up to $250 \mathrm{hPa}$ and the difference in vorticity between $850 \mathrm{hPa}$ and 250 $\mathrm{hPa}(850-250) \geq \xi_{\mathrm{V}}$ for some chosen value of $\xi_{\mathrm{V}}$. This implies a warm core.

(5) Criteria 3 and 4 must be achieved for at least $n$ consecutive time steps. 
Table 1. Calibration of parameters for TC identification based on ECMWF operational analyses and observed storms from Tropical Warning Centers

\begin{tabular}{lllrrr}
\hline $\begin{array}{l}\text { All } \\
\text { tropical } \\
\text { cyclones }\end{array}$ & $\begin{array}{l}\text { Hurricanes, } \\
\text { typhoons, } \\
\text { cyclones }>33 \mathrm{~ms}^{-1}\end{array}$ & $(6,6,4)$ & $(10,6,4)$ & $(12,6,4)$ \\
\hline 2003 & 75 & 33 & 69 & 48 & 39 \\
2004 & 72 & 36 & 78 & 52 & 41 \\
2005 & 80 & 38 & 82 & 62 & 48 \\
\hline
\end{tabular}

As can be seen, this is a simpler set of criteria that has been used in previous studies although several fields/levels are used. All that is required is the choice of values for the triplet $\left(\xi_{\mathrm{I}}, \xi_{\mathrm{V}}\right.$, $\mathrm{n})$. This requires calibration against observed tropical cyclones. Note, both criteria 3 and 4 could also be applied at full resolution, but would require a re-calibration. We have performed the calibration for $3 \mathrm{yr}$ of ECMWF operational analyses for 2003, 2004 and 2005 as this is considered the best available homogeneous data set dependent on observations. Table 1 shows the results for several choices of the triplet compared with best track data from the TWC. From this, the triplet $(6,6,4)$ was chosen as the best compromise for identifying the $\mathrm{TC}$ at this resolution. To illustrate the methodology, the tracks for the very active Atlantic year of 2005 are shown in Fig. 1 for the $(6,6,4)$ and $(12,6,4)$ selection criteria together with the individual track and further information for hurricane Katrina.

Figure 1 shows that the tracking and identification scheme is performing well identifying nearly all the observed TC. In addition, the specific example of hurricane Katrina is shown (Fig. 1c), which shows the whole track from first identification in the tropics at 2005/08/20:18, which is $2 \mathrm{~d}$ before it was first identified as a tropical depression, and into the mid-latitudes where it decays. The total lifetime is $\sim 20 \mathrm{~d}$ which is $12 \mathrm{~d}$ longer than the best track data, other storms have even larger differences depending on how long they take to grow and how far into the mid-latitudes they go. Comparing the track of Katrina in ERA40 with that of the observed track (not shown) shows that the two tracks are almost identical, in particular when the storm is classified as a hurricane. Figure 1d, e and f show the T63 vertical structure of Katrina, the dependence of the intensity (for vorticity) on resolution and the corresponding sea level pressure and maximum winds at full resolution, respectively, compared with those observed. Note, the differences in resolution have been obtained by spectral truncation and not new re-analyses. The vertical structure shows the typical growth of a tropical cyclone with a strong gradient in vorticity, in particular between 500 and $250 \mathrm{hPa}$ where the warm core is situated. This gradient can be seen to weaken and in fact reverse as the storm goes extra-tropical and attains a cold core before disappearing. The intensity, in terms of the vorticity shows a significant sensitivity to the resolution of the data with a large difference between the T63 and T159 resolutions and then an increase in going from T159 to T511 though perhaps not as dramatic as from T63 to T159, apart from the first 'spike' that occurs at day six after genesis. This appears to be due to a strong horizontal gradient in the vorticity field leading to a possible 'overshoot' in the interpolation used to find the maxima. This sensitivity to resolution is important when looking for changes associated with climate change, as will be discussed in our next paper, and is one reason why vorticity is a useful quantity for measuring intensity, though off coarse winds are more important when considering possible damage. The pressure and winds show much less sensitivity to the resolution with virtually no discernable difference between the pressure centre at T159 and T511. The winds show a little more sensitivity but not as much as the vorticity. Also, shown in Figure $1 \mathrm{f}$ are the observed values, which show a much deeper centre of pressure and higher wind speeds than the T511 resolution data. This type of diagnostics will be used throughout this paper.

\section{Structure and behaviour of modelled and assimilated TC}

We first show examples of TC from the model and ERA40 with similar characteristics. Figure 2a shows an example of a storm track, with the T159 vorticity as intensity, of an intense TC in ERA40 for the period 12 September-3 October 1991. The storm track agrees well with the observed Super Typhoon 21, although there are large differences in the intensity. The maximum wind speed in ERA40 is $45 \mathrm{~ms}^{-1}$, while the maximum wind speed for the Super Typhoon 21 is $66 \mathrm{~ms}^{-1}$. Furthermore, the observed maximum wind did not coincide with ERA40 but occurred a few days earlier. Figure 2c shows the evolution of vorticity at the 850,500 and $250 \mathrm{hPa}$, respectively. Around day six, there is an increase in the vertical vorticity gradient, indicating decreasing thermal wind due to the generation of a warm core. Around day 16 , the vertical vorticity gradient disappears as the vortex is transformed into an extra-tropical storm. The corresponding wind speed at $925 \mathrm{hPa}$ and surface pressure is shown in Figure 2e. There is no increase in the maximum wind speed at the time of the major intensification between day 15 and 16. A more detailed inspection of the wind field shows that it is becoming more homogeneous around the centre leading to the rapid increase in vorticity and reduction in central surface pressure.

Although there is a broad agreement in the number and distribution of TC between ERA40 and ECHAM5 during this period (Table 3) it is of course not possible to find any agreement in the evolution of individual TC in spite of the fact that the pattern of SST (according to the AMIP2 protocol) is the same in ERA40 and in ECHAM5, as the atmospheric circulation is only weakly constrained by the SST. Instead, we select a typical model generated TC that occurred during the same month but in a different year in the Western Pacific. Figure $2 b$ shows the track of the cyclone for the period 24th August-14th September 1987 with the intensity for the T159 vorticity. The vorticity intensity is 


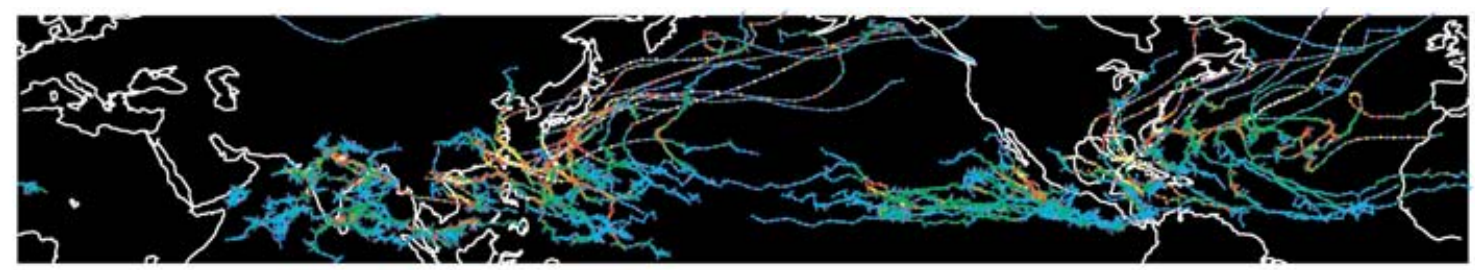

(a)

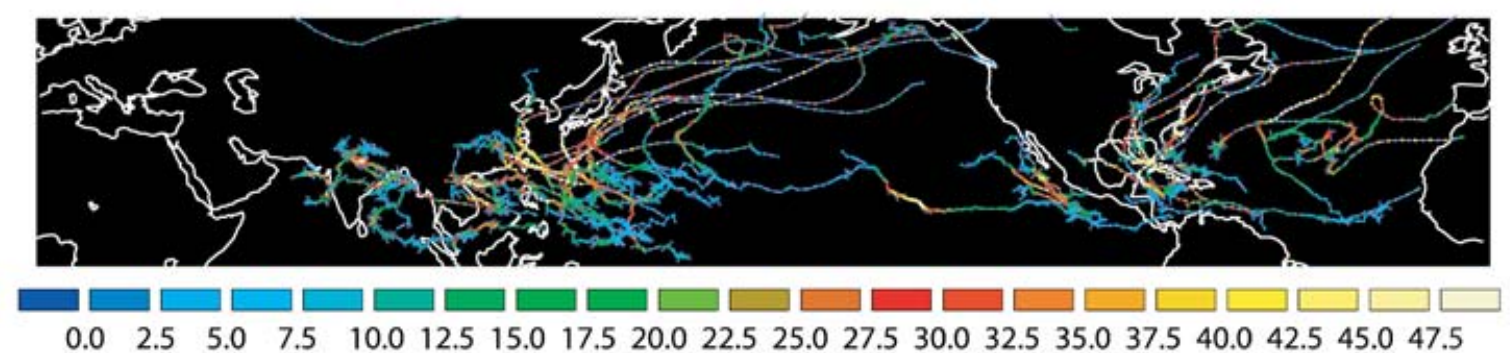

(b)



(c)

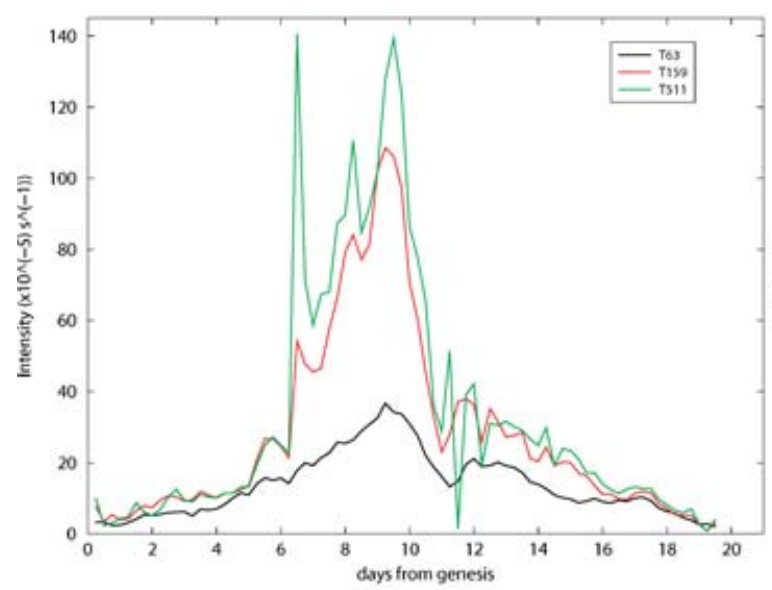

(e)

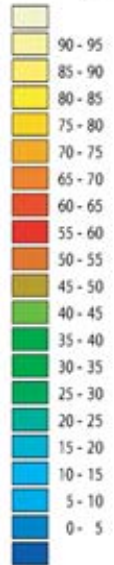

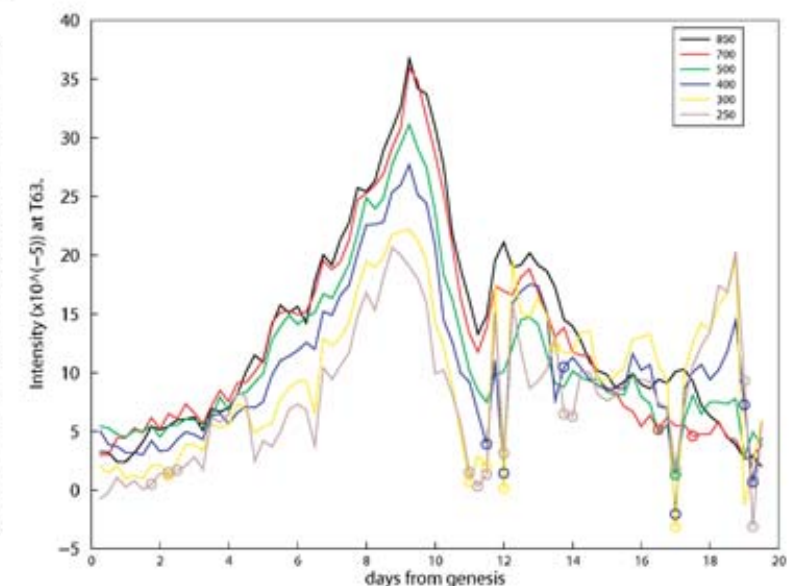

(d)

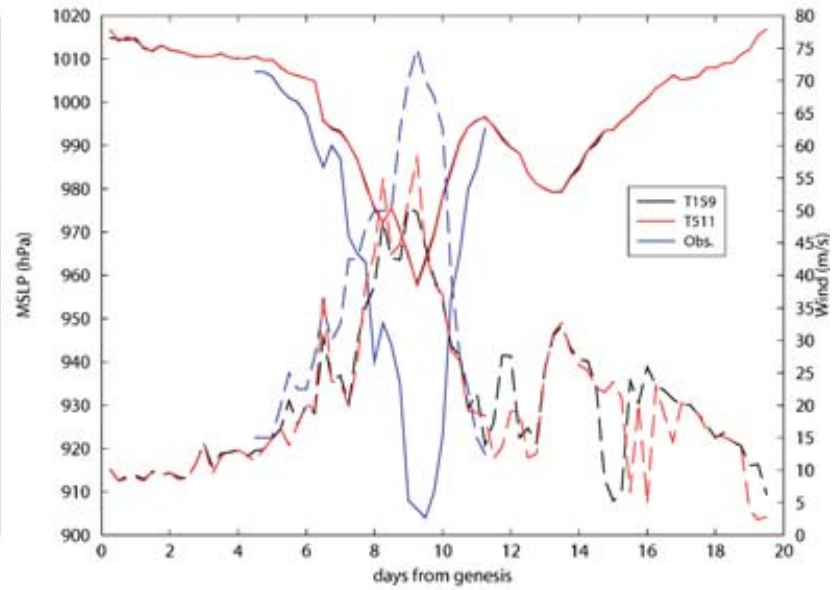

(f)

Fig. 1. Examples from ECMWF operational analyses for 2005, (a) identified TC using $(6,6$, 4), (b) identified TC based on $(12,6$, 4), (c) track of hurricane Katrina, first identified 2005/08/20:18, coloured dotes indicate the T159 intensities (vorticity $\times 10^{-5} \mathrm{~s}^{-1}$ ), at each 6 hourly position, (d) T63 vertical structure of hurricane Katrina, the open circles indicate values at the T42 centre at the relevant level due to failure to find a maximum, (e), impact of resolution on the intensity of Hurricane Katrina in terms of vorticity, (f) central pressure and maximum winds of hurricane Katrina compared with observations, full line is pressure and dashed line is wind speed. 


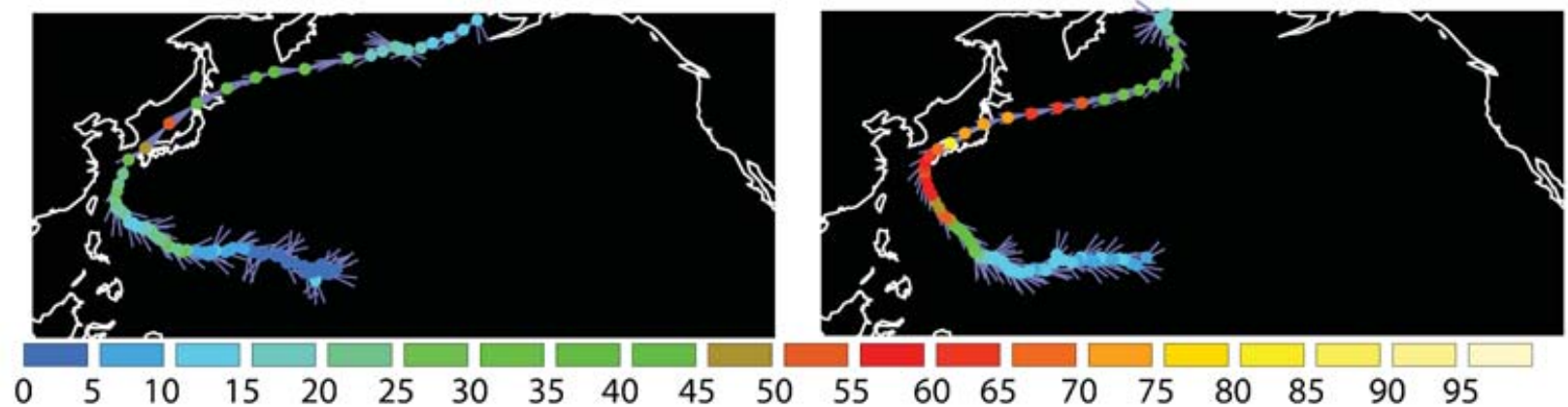

(a)

(b)

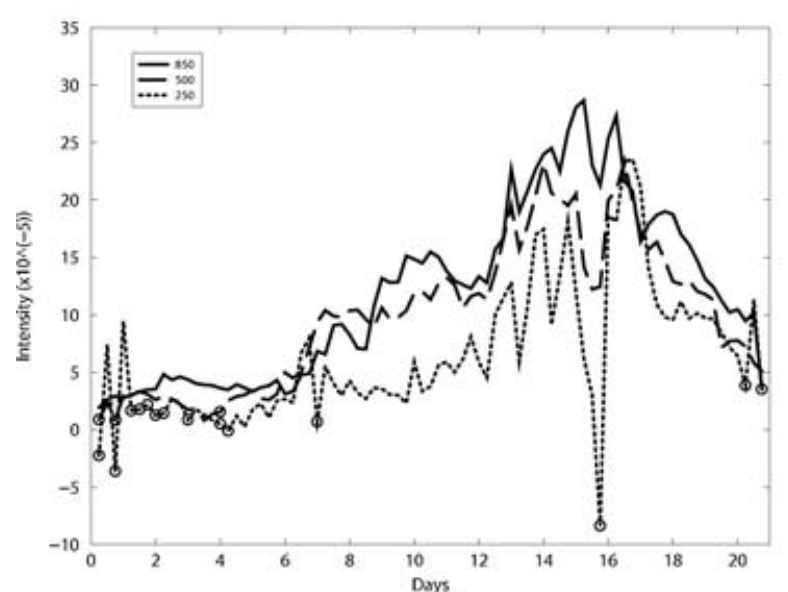

(c)

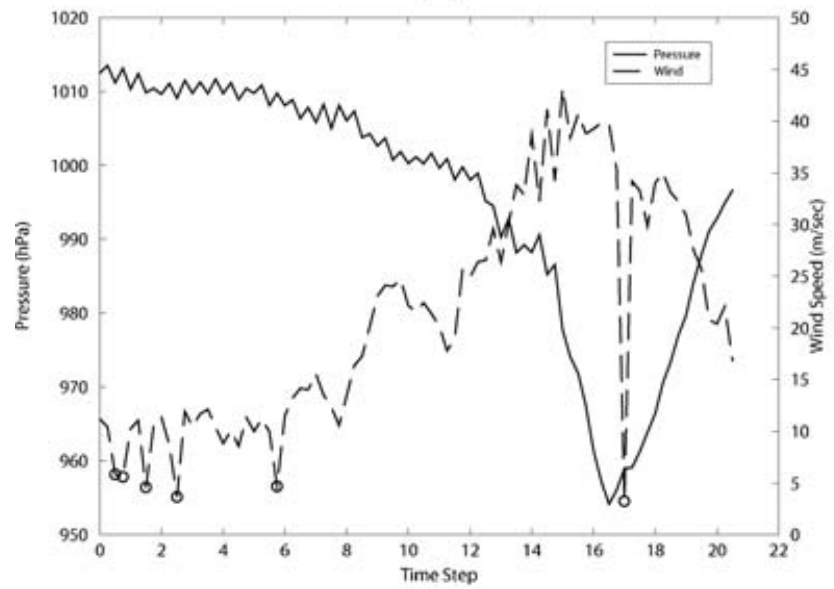

(e)

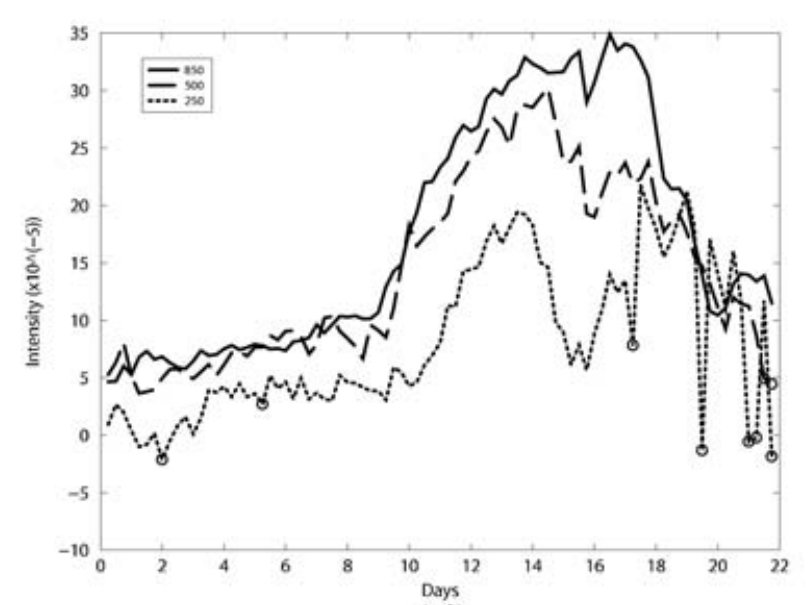

(d)

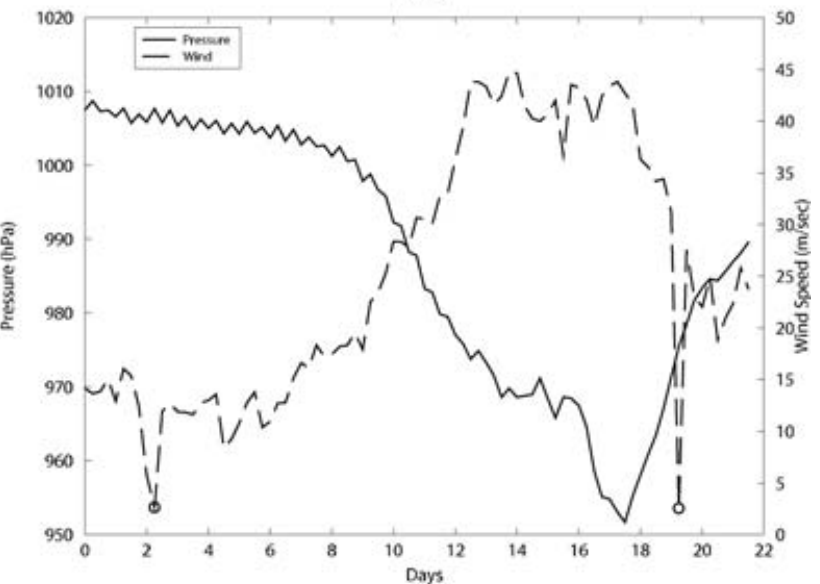

(f)

Fig. 2. Examples of similar storms from ERA40 and the ECHAM5 model. (a) Track of Super Typhoon 21 in ERA40, first identified 1991/09/12:18, intensity is vorticity at T159 $\times 10^{-5} \mathrm{~s}^{-1}$ (b) track of a similar storm in the ECHAM5 model, first identified 1987/08/24:06, intensity is vorticity at $\mathrm{T} 159 \times 10^{-5} \mathrm{~s}^{-1}$, (c) vertical structure of ERA40 storm, vorticity at 850, 500 and $250 \mathrm{hPa}$ at T63, (d) vertical structure of ECHAM5 storm, vorticity at 850,500 and $250 \mathrm{hPa}$ at T63, (e) MSLP and $925 \mathrm{hPa}$ winds for the ERA40 storm, (f) MSLP and $925 \mathrm{hPa}$ winds for the ECHAM5 storm. Note, open circles in (c)-(f) denote an extremum could not be found and values are at the T42 centre.

generally higher in the ECHAM5 storm than in the ERA40 one. The vertical structure, shown in Figure 2d is slightly different to the ERA40 storm with the vertical vorticity gradient being larger, associated with a more marked warm core. We have inspected a few other storms suggesting that this could be a systematic difference between ECHAM5 and ERA40. (JRA25 also appears to have more marked warm cores than ERA40 being more similar to ECHAM5, see later where composites are discussed). Further 
Table 2. Summary of the number of TC per year as a function of maximum wind speed

\begin{tabular}{lccc}
\hline & $>18 \mathrm{~ms}^{-1}$ & $>33 \mathrm{~ms}^{-1}$ & $>50 \mathrm{~ms}^{-1}$ \\
\hline ECHAM5 T159 & 94.4 & 34.6 & 3.7 \\
ERA40 & 67.5 & 14.2 & 0.05 \\
JRA25 & 58.5 & 15.3 & 0.1 \\
Observed & 58.9 & 32.9 & 15.7 \\
\hline
\end{tabular}

Table 3. Summary of the number of TC per year as a function of maximum intensity in vorticity at $850 \mathrm{hPa}$

\begin{tabular}{lcccc}
\hline & All $(6,6,4)$ & $>2 \times 10^{-4}$ & $>5 \times 10^{-4}$ & $>1 \times 10^{-3}$ \\
\hline ECHAM5 T159 & 96.8 & 86.8 & 28.8 & 1.2 \\
ERA40 & 80.2 & 72.1 & 1.9 & 0 \\
JRA25 & 61.8 & 44.3 & 0.33 & 0 \\
\hline
\end{tabular}

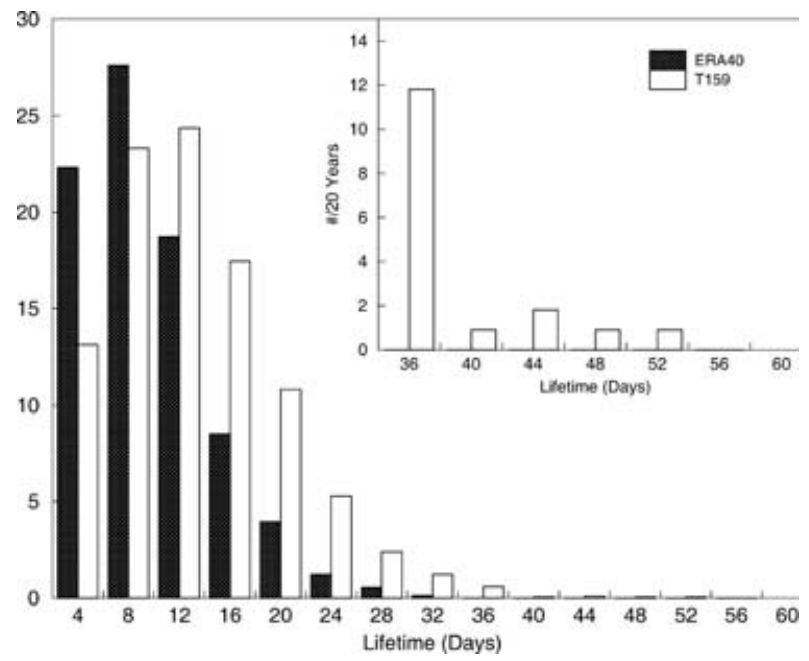

Fig. 3. Lifetime histograms for ERA40 and ECHAM5. Inset shows the tail scaled to a 20 -yr period.

evaluation on this will be presented in the discussion. The behaviour of the minimum pressure and $925 \mathrm{hPa}$ winds in general indicates a more confined structure of the TC though for this particular storm, shown in Figure 2f, the $925 \mathrm{hPa}$ wind speed and surface pressure are similar to the ERA40 storm. However, as will be discussed later the TC in ECHAM5 are generally stronger than in ERA40 (see Table 2 and Table 3).

We follow each individual storm during its lifetime even before and after it has lost its typical structure that qualifies the storm as a TC. The storms can at many times exist as a weak vortex for many days until it suddenly undergoes a development into a TC. At the end of its lifetime it is mostly transformed into an extra-tropical storm as it is caught up in the westerly winds at middle latitudes. Figure 3 shows the distribution of the lifetime of the individual storm tracks for ERA40 and ECHAM5, respec- tively. The result is comparable for ERA40 and ECHAM5, as we use the same criteria for the identification of the TC, but the TC are generally more long-lived in ECHAM5. The lifetime of the observed (best track) storms are shorter (not shown) as they have been identified with different criteria and do not generally include the weak initial phase nor the phase as extra-tropical storms. There are huge variations in the lifetime from the minimum of $2 \mathrm{~d}$ to a maximum of more than $30 \mathrm{~d}$ in ERA40 and more than $50 \mathrm{~d}$ in ECHAM5. There is also a relation between the maximum intensity and lifetime and stronger storms are active for a longer time than weaker storms, but with a large variability.

As described in the previous sections, we have calculated all the tropical warm core eddies in ERA40 and in ECHAM5 for the period 1978-99. Table 2 summarizes the number of vortices, for the period 1978-99, with a maximum wind speed higher than $18 \mathrm{~ms}^{-1}$, higher than $33 \mathrm{~ms}^{-1}$ and higher than $50 \mathrm{~ms}^{-1}$, respectively. The first column in Table 2 shows the total number of tropical cyclones per year, including tropical depressions, the second column the number of category 1 (on the Saffir-Simpson scale) and higher storms per year and the last column indicates the number of storms of category 3 and higher. We first note that ERA40 and even more so the ECHAM5 have more eddies than the observations. The main reason for this is probably the somewhat arbitrary criteria used to determine a warm core vortex, here having a minimum difference in vorticity between 850 and $250 \mathrm{hPa}$ of $6 \times 10^{-5} \mathrm{~s}^{-1}$. Sensitivity studies have shown that the number of storms are sensitive to the value of the minimum difference in vorticity at the two levels (c.f. Table 1). Another explanation could be due to differences in lifetime of the eddies. Finally, it may well be the case that the operational observing systems may have underestimated the number of tropical storms in remote ocean regions though they could also have overestimated the wind speeds used as a measure of intensity. These type of errors are more likely to occur in earlier periods than the data used here. In addition, the differences in the averaging periods of the wind speeds may also have an impact on these differences, though this is difficult to ascertain.

The number of TC fulfilling the criteria for a category 1 or stronger storm $\left(>33 \mathrm{~ms}^{-1}\right)$ are rather similar in ECHAM5 and in the observations, while ERA40 has less than half as many TC in this category. This can clearly be seen from Figure 4 which shows the tracks of all storms that attain a maximum wind speed greater than $33 \mathrm{~ms}^{-1}$. This shows that in ERA40 there are hardly any storms in the Eastern Pacific that fulfil this criteria (this also occurs in JRA25, see later) as well as the Atlantic appearing somewhat reduced in activity compared with the observations. ECHAM5 on the other hand, does have numerous systems in the Eastern Pacific that fulfil this criteria, though the Atlantic still appears lacking and the Indian Ocean is far too active (see later). Finally for the most powerful vortices with a wind speed stronger than $50 \mathrm{~ms}^{-1}$, corresponding to a category 3 and higher, there is only one storm in a 20-yr period in ERA40, while ECHAM5 has 74. The observed intense storms with category 3 or higher are 


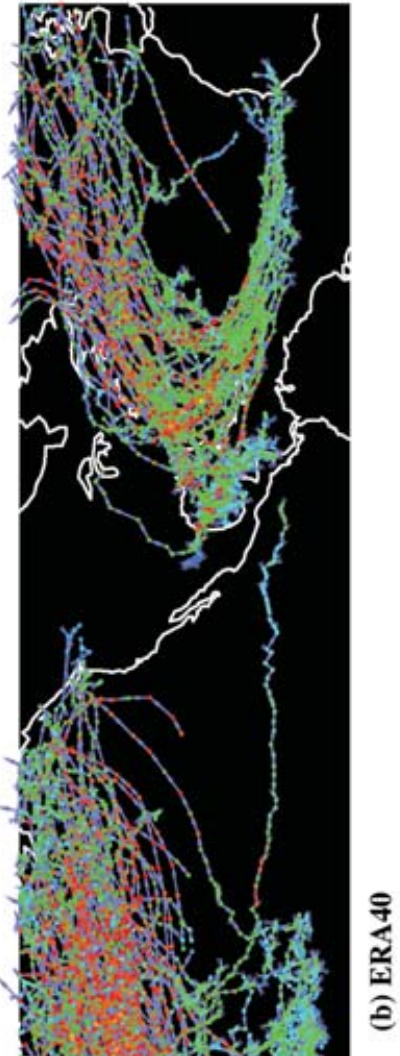

Nives

rit.

in:

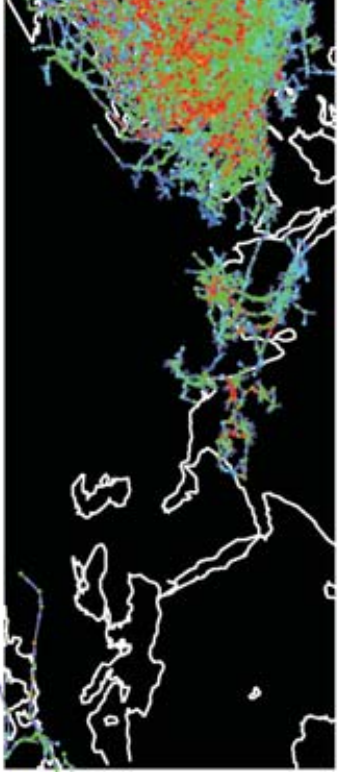

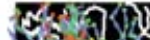

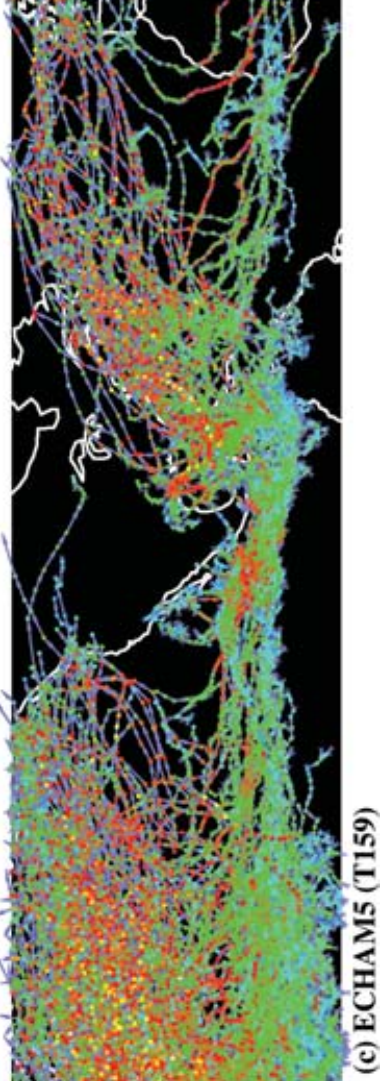

Bivis

If is

if.

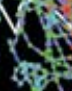

an:




four times more common in the observations than in ECHAM5. Preliminary experiments with a model at higher resolution than T159 indicate that more powerful storms are more common and similarly experiments with lower resolution show a clear reduction in the number of intense storms. We therefore suggest that the lower number of intense storms in ECHAM5 compared to observations is mainly a consequence of insufficient model resolution.

However, the smaller number of powerful storms in ERA40 is not only due to resolution, as ERA40 actually has the same horizontal resolution as ECHAM5. Furthermore, we have evaluated the storms on the same linear grid consisting of $480 \times 240$ points per horizontal field. The difference in handling intense storms can also be seen in Table 3 comparing the number of storms as a function of the $850 \mathrm{hPa}$ vorticity. As the vorticity increases, the number of storms in ERA40 is rapidly reduced compared to ECHAM5. There are several possible explanations why ERA40 has a smaller number of intense vortices. This includes the consecutive interactions of model information and contributions from observations, where it is likely that there are insufficient observations to support the evolution of an intense vortex in several ocean areas. A small number of observations might thus pose problems for the data assimilation in particular if based on 3-D Var where it may be difficult to maintain the delicate balance between wind field, mass field and convectively driven latent heat source in an intense tropical storm. It may be expected that an assimilation based on 4D Var would perform differently and presumably better preserve such intense tropical vortices. The example of Katrina shown in Figure 1 appears to support this, since the operational analysis uses 4D Var.

\section{Geographical distribution}

We first show distributions for genesis and track density computed from the cyclone tracks for ECHAM5, ERA40 and observations using the spherical kernel method (Hodges, 1996) as used in other studies (Thorncroft and Hodges, 2001; Hodges et al., 2003, Bengtsson et al., 2006).

We show first the densities of cyclogenesis in Figure 5, for the TWC best track data (Fig. 5a), followed by the densities for ERA40 and ECHAM5 (Fig. 5b and c), respectively, based on the full tracks (see later). We define cyclogenesis as the point where we can first identify a vortex at $850 \mathrm{hPa}$ with a vorticity of $5 \times 10^{-6} \mathrm{~s}^{-1}$. We select all TC that fulfil the criteria $(6,6,4)$ as defined in section 3 . With these conditions, the total number of storms in ECHAM5 and ERA40 are roughly the same (Table 3). The observed storms as compiled by TWC are not selected according to strict criteria due to lack of quantitative observations in remote areas, and the number of observed storms are less than the analyzed TC in ERA40 (Table 3).

As can be seen in Figure 5a for the TWC data set, the most active and concentrated area of cyclogenesis occurs in the Eastern Pacific with its centre some $500 \mathrm{~km}$ southwest of the Mexican coast. The area of cyclogenesis in the Western Pacific is more extended into the central Pacific but with an additional maximum in the South China Sea. In the Northern Indian Ocean, the dominant part of the cyclogenesis occurs in the Bay of Bengal. However, it is interesting to note that the cyclogenesis in the Atlantic Ocean has only weakly marked centres and is more widely distributed. It should also be noted that the cyclogenesis in the observations will differ from those in ERA40 and ECHAM5 since the observations only consist of data for when the storm is first classified as a tropical depression, whereas the ERA40 and ECHAM5 data consist of storms with earlier stages of their development included.

The cyclogenesis areas in ERA40, shown in Figure 5b, are rather similar to the observations except for an area at the African Atlantic coast that has been noted before for easterly wave activity (Thorncroft and Hodges, 2001). There is also higher activity in the Northern part of the Bay of Bengal. However, this may be because some monsoon depressions are classified as TC. The large genesis seen in the Eastern Pacific is perhaps surprising when considered with Figure 4, but this just highlights that most of these storms are quite week having maximum winds $<33 \mathrm{~ms}^{-1}$.

For ECHAM5, shown in Figure 5c, there is a marked band of cyclogenesis in the Pacific Ocean but broadly a distribution similar to ERA40. The largest differences are in the Atlantic where the model has an intense centre of cyclogenesis North of Panama. The cyclogenesis region in the Bay of Bengal is also more pronounced in ECHAM5. We believe some of the differences in Central America between ECHAM5 and to some extent even with ERA40 with the TWC data set is probably due to model resolution, as the sharp mountain areas in Central America cannot be properly resolved by the model. This has the effect that some storms generated in the Southern Caribbean Sea could find their way into the Pacific, which is less common in reality. We intend to consider this in more depth in the second paper (Bengtsson et al., 2007) where we will be using a higher resolution than here.

However, the area of genesis in the observations are more dependent on the identification criteria than those shown for ERA40 and ECHAM5 since the observations only show the storms once they have been classified as TC, whereas in ERA40 and ECHAM5, the whole lifecycle is captured from very weak initial vortex. To explore the impact on the genesis plots of restricting the ERA40 and ECHAM5 tracks to only those parts of the tracks when the storms are classified as TC, we have recalculated the genesis statistics using the parts of the tracks that satisfy the conditions $\xi_{\mathrm{I}} \geq 6 \times 10^{-5} \mathrm{~s}^{-1}$ and $\xi_{\mathrm{V}} \geq 6 \times 10^{-5} \mathrm{~s}^{-1}$ at T63, this is equivalent to winds $\geq 18 \mathrm{~ms}^{-1}$. The new genesis plots for ERA40 and ECHAM5 are shown in Figure 5d and e, respectively. For ERA40, the change in the distribution is not particularly large but does show a reduction in the Eastern Pacific and a shift westwards in the Western Pacific and perhaps is more similar to the observations. For ECHAM5, the changes 


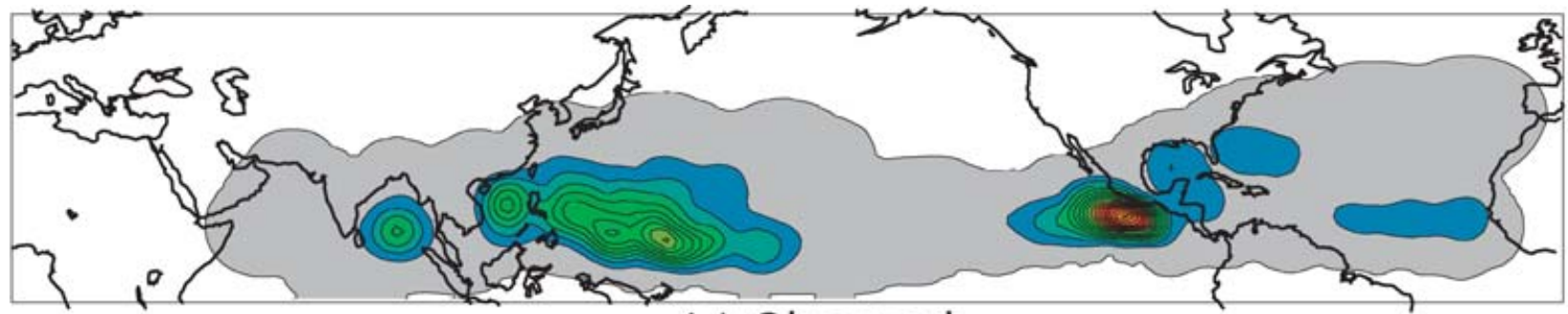

(a) Observed

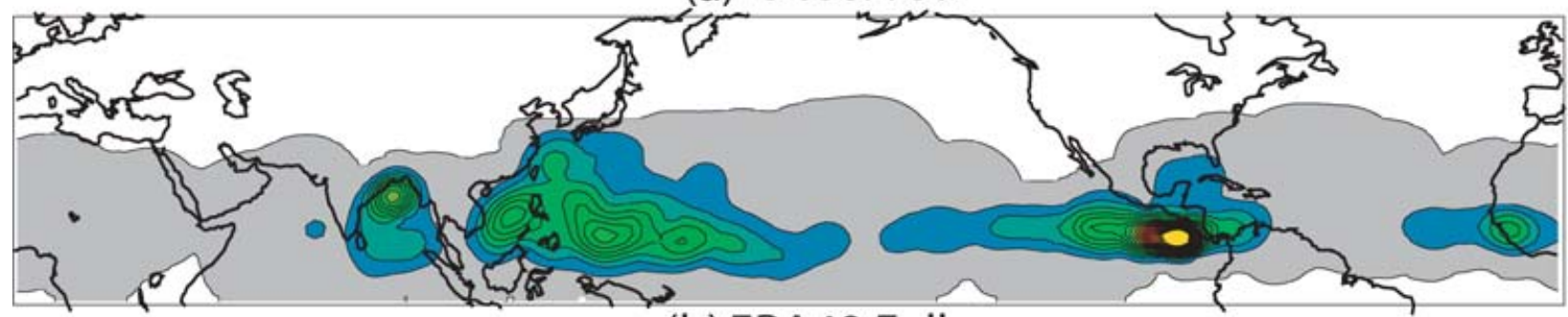

(b) ERA40 Full

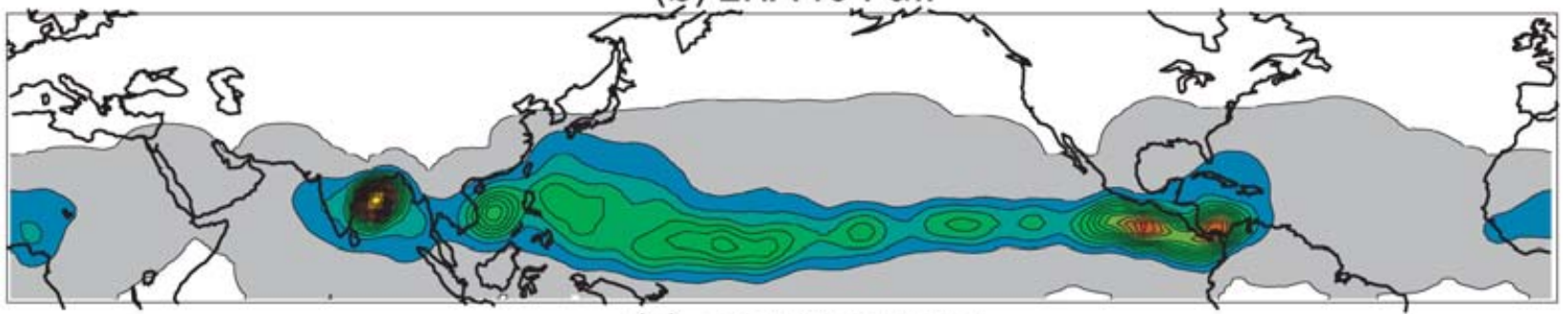

(c) ECHAM5 FULL

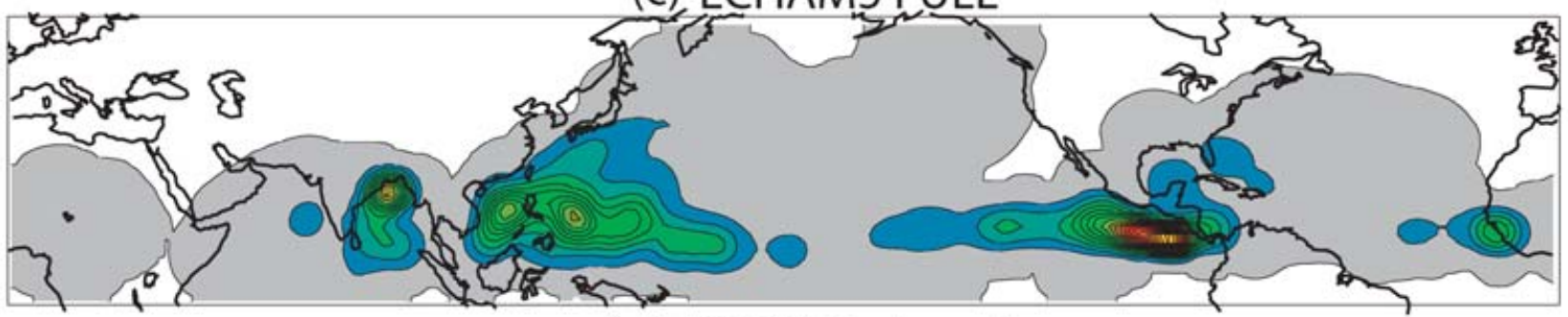

(d) ERA40 Reduced



(e) ECHAM5 Reduced

$\begin{array}{llllllllllllllllllll}0.0 & 0.5 & 1.0 & 1.5 & 2.0 & 2.5 & 3.0 & 3.5 & 4.0 & 4.5 & 5.0 & 5.5 & 6.0 & 6.5 & 7.0 & 7.5 & 8.0 & 8.5 & 9.0 & 9.5\end{array}$

Fig. 5. Genesis density as number density per year per unit area where the unit area is equivalent to a $5^{\circ}$ spherical cap $\left(\sim 10^{6} \mathrm{Km}^{2}\right)$. (a) Observations, (b) ERA40 full tracks, (c) ECHAM5 full tracks, (d) ERA40 reduced tracks, (e) ECHAM5 reduced tracks. (d) and (e) are based on the tracks reduced in length so as to satisfy the identification criteria, $\xi_{\mathrm{I}} \geq 6.0 \times 10^{-5} \mathrm{~s}^{-1}, \xi_{\mathrm{V}} \geq 6.0 \times 10^{-5} \mathrm{~s}^{-1}$ only. 




(a) Observed

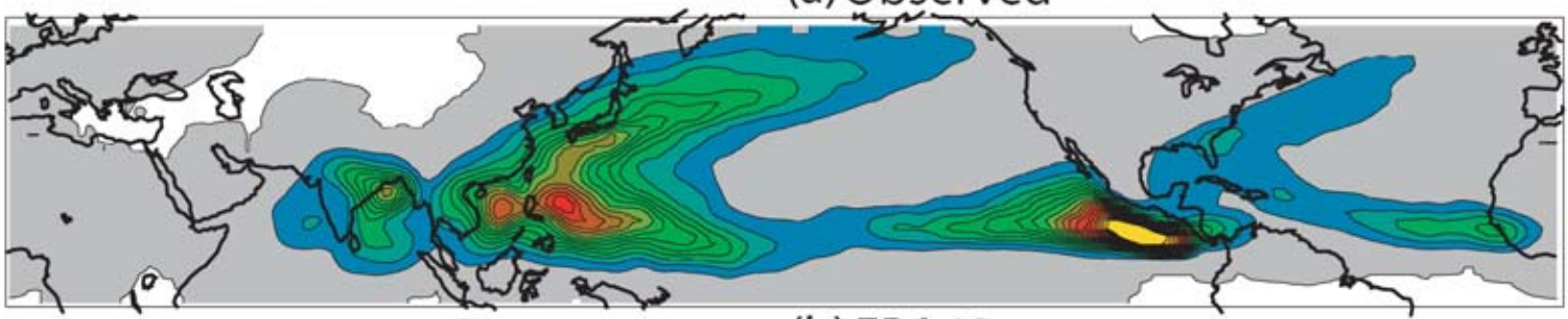

(b) ERA40

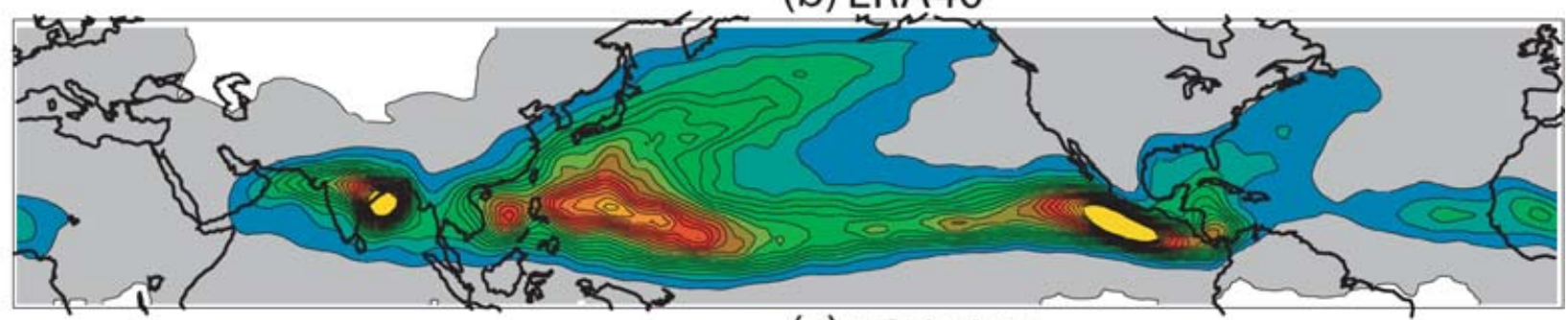

(c) ECHAM5



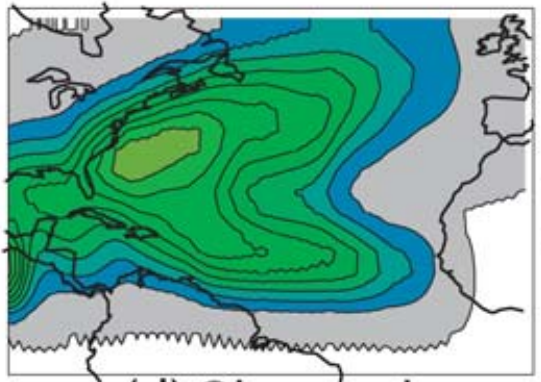

(d) Observed

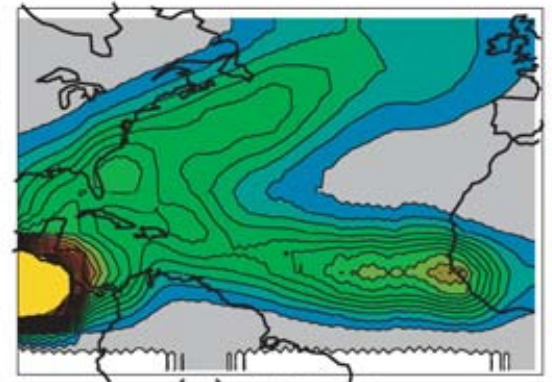

(e) ERA40

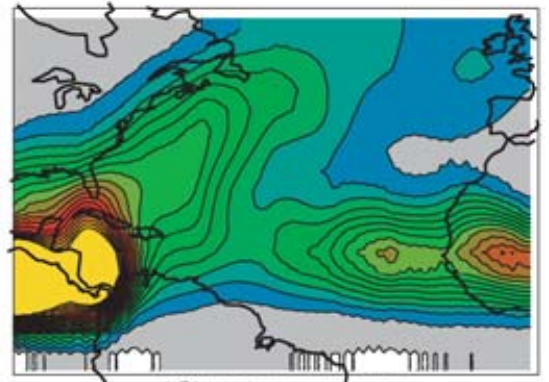

(f) ECHAM5

\section{$\begin{array}{llllllllllllllllllll}0.0 & 0.5 & 1.0 & 1.5 & 2.0 & 2.5 & 3.0 & 3.5 & 4.0 & 4.5 & 5.0 & 5.5 & 6.0 & 6.5 & 7.0 & 7.5 & 8.0 & 8.5 & 9.0 & 9.5\end{array}$}

Fig. 6. Track density as number density per year per unit area where the unit area is equivalent to a $5^{\circ}$ spherical cap $\left(\sim 10^{6} \mathrm{Km}^{2}\right)$. (a) $\mathrm{Observations,}$ (b) ERA40, (c) ECHAM5, (d) Observations for the Atlantic, (e) ERA40 for the Atlantic, (f) ECHAM5 for the Atlantic. (b), (c), (e) and (f) are based on the $(6,6,4)$ identification criteria.

are much larger with a reduction in activity through the central Pacific, and more concentrated activity in the Eastern Pacific with a corresponding reduction of the Central American genesis. The largest change is the shift westwards and enhancement of the Western Pacific genesis, which is now much closer to observations. The changes in the Eastern Pacific/Central America reinforce the argument that when the entire tracks are used there are more systems coming through from the Caribbean into the Eastern Pacific than is observed.

In Figure 6a-c, we investigate the track density. Note that, although there are more storms in ECHAM5 and ERA40 compared to the observed storms (Table 3 ), there are nevertheless considerable similarities in the patterns of the track density. However, as with the genesis, the track density computed with the full tracks 
in ERA40 and ECHAM5 will differ from the observed track density due to the different identification criteria used for the observations. The most direct comparison can be made between ERA40 and ECHAM5 since the tracks have been obtained in the identically same way. This shows a remarkably similar distribution between the two though with ECHAM5 having larger values for the track density stretching across the Pacific. There are several possibilities for this, one is the fact that the propagation of the storms is likely to be smoother in the model than in the re-analysis resulting in longer tracks, particularly when the storm is in its weaker stages. Another possibility is the fact that the storms are generally more intense in the model allowing them to be tracked for longer than in the re-analysis. As discussed above, this could be due to problems with the data assimilation used in ERA40. Also note, the relatively low levels of the density in the Atlantic compared with the other ocean basins. To enable a better comparison to be made in the Atlantic, the density for this region is plotted separately in Figure $6 \mathrm{~d}-\mathrm{f}$. This shows that ERA40 and the observations look remarkably similar apart from the fact that in ERA40 we are identifying them earlier so that the 'storm track' extends back further over Africa. For ECHAM5, this also has a 'storm track' extending from Africa, but there is a marked reduction in track density in the mid-Atlantic in contrast to the observations and ERA40.

The most apparent difference in the track density is in the North Indian region where the model generates an intense track of eddies over the Northern sub-Indian continent that is not found in the observed data set. ERA40 also appears to have more activity in this region than the observations. As discussed elsewhere, this is related to the interpretation of monsoon depressions and the tendency of the model to generate too intense monsoon depressions.

In a similar vein to the genesis density, we also computed the track density for just those parts of the tracks that are classified as TC for ERA40 and ECHAM5 (not shown). This produced distributions that were more similar to those of the observed track density as was the case for the genesis density.

We have also compared the geographical variation of intensity of vortices as well as their deepening rate with the observations. Although the maximum intensity is underestimated in ECHAM5, and even more so in ERA40, the regions where the maximum intensity is attained appear very similar (not shown). Similarly, the model underestimates the deepening rate. We suggest this is due to insufficient resolution and the use of the primitive equations (e.g. Krishnamurti et al., 2005). The same is the case for ERA40 suggesting that insufficient observations are available to obtain the correct deepening rate. Lacking observations ERA40 has the same restrictions as the model.

In Figure 7a, we show the observed number of storms as a function of the maximum attained wind speed in the four TC regions, Atlantic, Eastern Pacific, Western Pacific and the Northern Indian Ocean. The largest number of observed storms occurs in the Western Pacific, where the most intense storms occur with
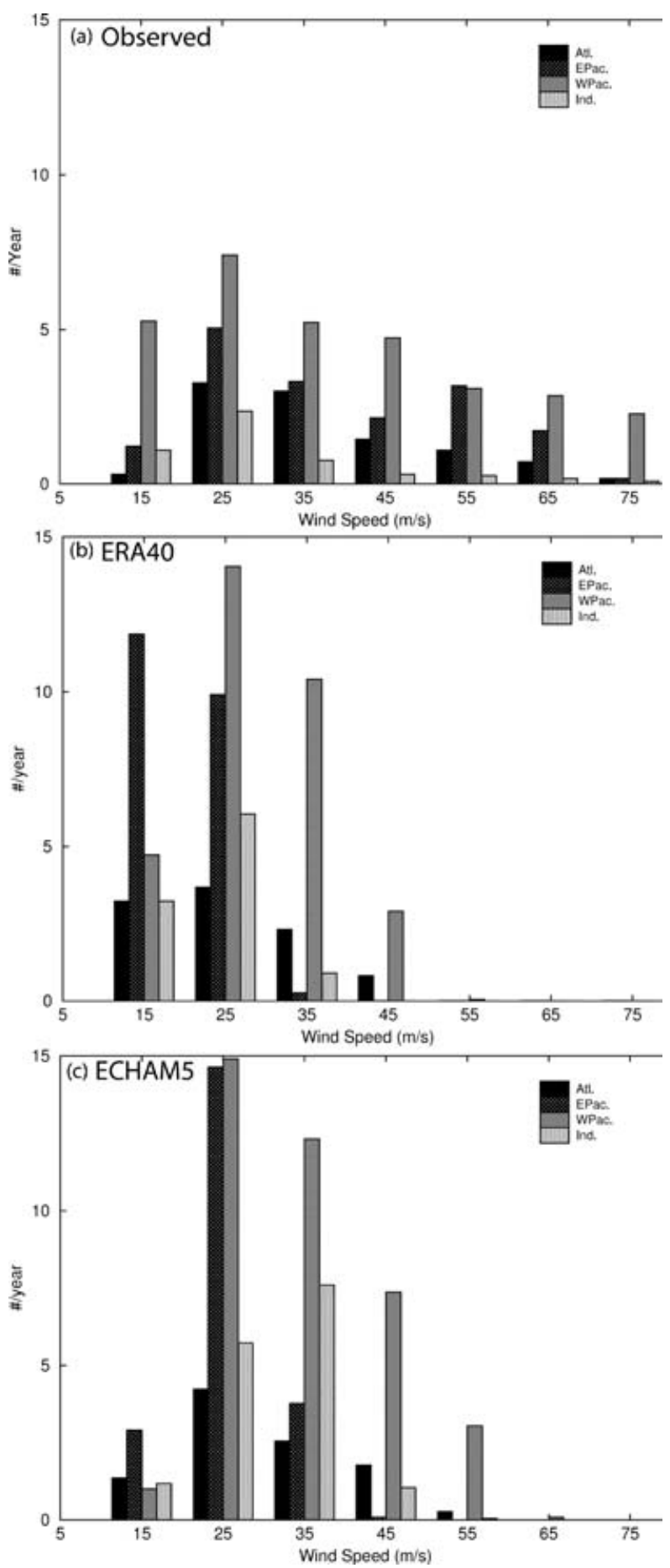

Fig. 7. Distributions of maximum attained wind speeds in different ocean basins for (a) Observations, (b) ERA40 and (c) ECHAM5.

sustained maximum wind speeds of more than $75 \mathrm{~ms}^{-1}$. The Eastern Pacific has the second largest number of storms. The distribution has a double structure with a proportionally high number of storms in the range $50-60 \mathrm{~ms}^{-1}$. The number of TC 


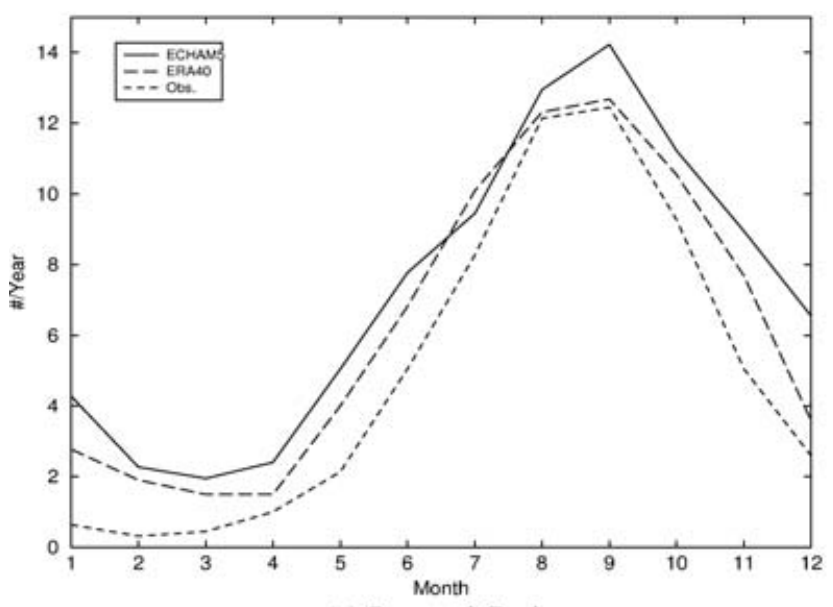

(a) Seasonal Cycle

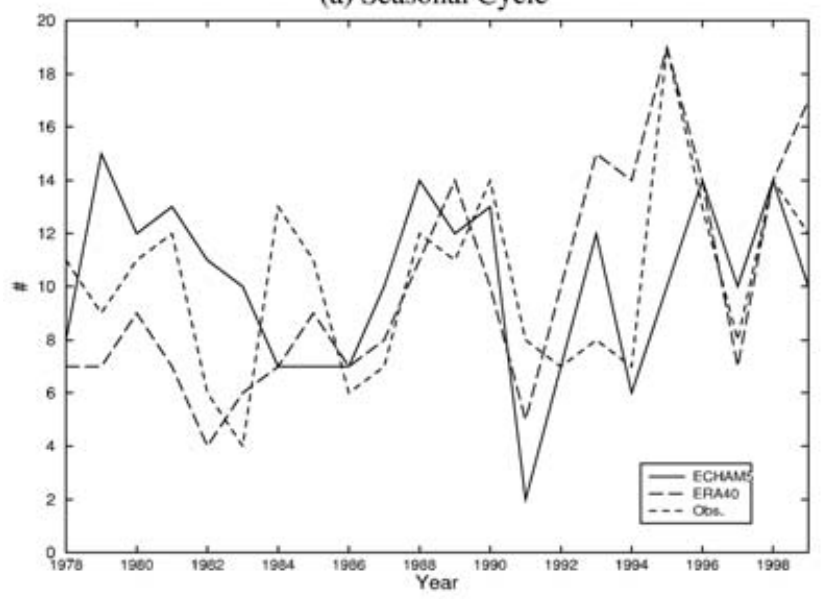

(c) Annual Variability (Atlantic)

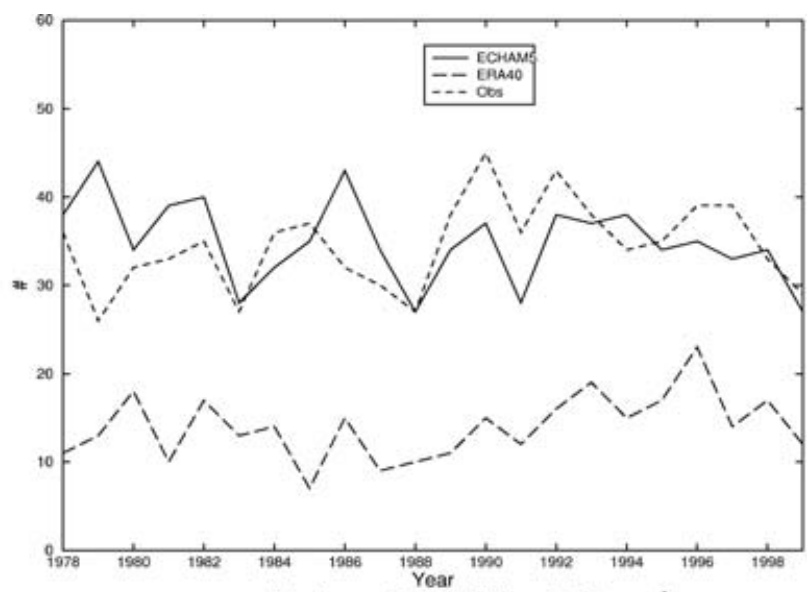

(b) Annual Variability $\left(>33 \mathrm{~ms}^{-1}\right)$

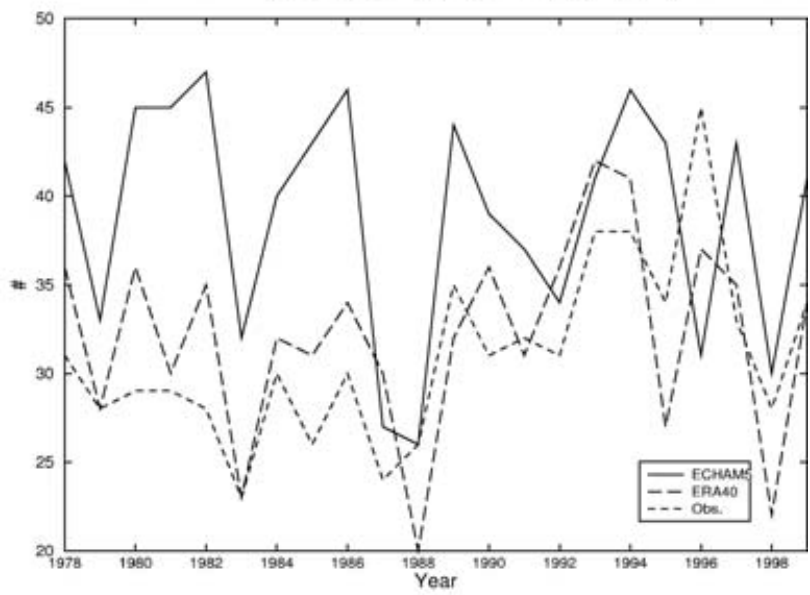

(d) Annual Variability (W. Pacific)

Fig. 8. Seasonal and annual variability of storms based on the $(6,6,4)$ identification criteria for ECHAM5, ERA40 and Observations. (a) Seasonal cycle, number per year, (b) annual variability in numbers of storms that have maximum wind speeds greater than $33 \mathrm{~ms}^{-1}$, (c) annual variability in the Atlantic, all storms and (d) annual variability in the Western Pacific, all storms.

in the Atlantic are less than in the two Pacific regions, but they can occasionally be very powerful storms. The North Indian Ocean has the smallest number of storms. In ERA40, the number of TC is greater than in the observations but in all regions with a distribution towards proportionally less intense storms (Fig. 7b). This is particularly the case in the Eastern Pacific where few storms above $33 \mathrm{~ms}^{-1}$ are found. There are also more storms in the North Indian Ocean than in the observations with an average of six storms a year reaching wind speeds of $20-30 \mathrm{~ms}^{-1}$ , whereas the observed data set only indicates two storms in this range. Finally, ECHAM5 has the largest number of storms in all regions except the Atlantic where the number is the same as in ERA40 (Fig. 7c). There are a proportionally large number of strong storms in the North Indian Ocean. As will be discussed later, it is suggested that this is due to the fact that ECHAM5 predicts too intense monsoon depressions, which are then classified as TC. Finally, the ECHAM5 underestimates the very intense $\mathrm{TC}$ in all regions but less so than in ERA40.

\section{Interannual variability and possible large scale processes controlling the TC}

There is an overall agreement in the seasonal distribution of TC between the observations, ERA40 and ECHAM5 all having a maximum in September, as shown in Figure 8a for the seasonal cycle. The seasonal distribution is somewhat more pronounced in the observations with hardly any storms in the winter months. However, a more detailed inspection indicates regional differences (not shown). Both observations and ERA40 show an early maximum in the Eastern Pacific in July or August, while ECHAM5 has a flat distribution with storms developing into late autumn. At the same time there is a later maximum in the Atlantic in ECHAM5 than both observations and ERA40. The largest difference is in the North Indian Ocean with a flat distribution from June through September in ECHAM5 that is different from the observed maximum before and after the monsoon. There is a similar distribution in ERA40 as in ECHAM5 but less pronounced. We suggest that this difference could partly be 
due to differences in the classification of TC in observations and in ERA40 and ECHAM5 as used here and partly because of a tendency by the model to over predict the intensity of monsoon depressions.

The number of identified observed tropical storms in the period 1978-1999 varies between 52 in 1983 and 79 in 1992 with an average value of 65 . The period is too short to identify a trend but there are more storms in the 1990s than in the 1980s. However, for storms with a maximum intensity of more than $33 \mathrm{~ms}^{-1}$, shown in Figure 8b, there is no noticeable difference between the 1980s and 1990s and no obvious trend throughout the period. This is also true for both ECHAM5, which shows a very similar number of storms to observed for this criteria (c.f. Table 2), and ERA40, which shows a much lower level of activity. Correlation between the de-trended time series are relatively low with values of $0.23,0.33$ and 0.38 for correlations between observations and ERA40, observations and ECHAM5 and ERA40 and ECHAM5, respectively.

As discussed above, the number of tropical storms identified in ERA40 and ECHAM5 is higher than in the observations, particularly so in the Indian Ocean, though the number of intense storms is less. We discuss here in more detail the interannual variability of storms in the Atlantic and in the Western Pacific. Figure 8c shows the variability of storms in the Atlantic for all storms. The number of observed storms in observations and ERA40 (using $6,6,4$ ) broadly follow each other with the minimum of Atlantic storms in 1997 and the maximum in 1995 being very similar. In other years there are larger differences as in 1992 and 1993. There are also large differences between ECHAM5 and observations, but generally not much worse than between ERA40 and the observations. Correlation between the de-trended time series are $0.57,0.35$ and 0.38 for correlations between observations and ERA40, observations and ECHAM5 and ERA40 and ECHAM5, respectively. However, as will be discussed below, there is a consistent response to ENSO in reasonable agreement with observations.

The agreement with ERA40 and observations is better in the Western Pacific than in the Atlantic, as shown in Figure 8d, for all storms. Note, for example the high number of storms in 1996 and the low number in 1998. There is also a good agreement in the low-frequency variation. ECHAM5 is here significantly worse with a larger number of storms as well as a larger interannual variability. There is agreement in some years, such as in 1983, 1987, 1988 and the 1998 minimum, but it is overactive in years with more storms than normal. Correlation between the de-trended time series are $0.69,0.23$ and 0.0 for correlations between observations and ERA40, observations and ECHAM5 and ERA40 and ECHAM5, respectively.

More generally, the interannual variation of storms with a maximum attained wind speed larger than $33 \mathrm{~ms}^{-1}$, as shown in Figure 8b, indicates the agreement between observations and ECHAM5 is better for storms satisfying this criteria. However, the agreement in total number and annual variability is possibly fortuitous as the number of weaker storms in ECHAM5 is higher and the number of more intense storms are less than in observations (see Table 2). The agreement with ERA40 is poor as the number of the more intense storms in ERA40 is significantly less than both observations and ECHAM5.

We have also investigated the relation in ECHAM5 between the SST anomalies, the large-scale divergent flow and the vertical wind shear with the interannual variability of TC in the Atlantic and in the Western Pacific, respectively. For the Atlantic, we take the difference between the representative years of high activity in ECHAM5 (1988 and 1996) and the years of low activity (1991 and 1997). Years are chosen from the latter period where we believe the SST's are more reliable. The corresponding SST difference for the composite years can be seen in Figure 9a. This shows a marked cold anomaly in the central and Eastern equatorial Pacific but higher temperatures than normal in the tropical Atlantic and Western Pacific. The difference in the $850 \mathrm{hPa}$ velocity potential (Fig. 9c) has a marked centre of subsidence over the central Pacific and areas of large-scale ascending motion over the Western Pacific and Indonesia as well as over the central and eastern Atlantic. We suggest that such a circulation pattern would favour development of tropical cyclones in the regions of the tropical Atlantic (and Western Pacific). Tropical cyclone studies are in agreement that a strong vertical wind shear is detrimental to the development of tropical cyclones. Figure 9e shows the difference of the composite years in the vertical wind shear (computed between 250 and $850 \mathrm{hPa}$ ) for the Atlantic region, this shows a distinct decrease over the tropical Atlantic and the West Indies in the high-activity years compared with the low-activity years consistent with the importance of the wind shear as a contributing factor to TC activity.

For the Western Pacific, we take the difference between the years of high activity in ECHAM5 (1986 and 1994) and the years of low activity (1988 and 1998). The corresponding SST difference can be seen in Figure 9b. The pattern is in several respects the opposite of the SST difference in Figure 9a with a warm anomaly in the central equatorial Pacific but with lower temperatures than normal in the tropical Atlantic and Western Pacific. The difference in the $850 \mathrm{hPa}$ velocity potential (Fig. 9d) is also practically the reverse of the pattern in Figure 9c having a marked centre of ascending motion over the central Pacific and areas of large-scale subsidence over the Western Pacific and Indonesia as well as over the central and Eastern Atlantic. Such a circulation pattern would favour transport of surface air into the region of the tropical Pacific where tropical storms normally develop. Similarly there are favourable vertical wind shear in the region where Pacific eddies develop into tropical cyclones (Fig. 9f). So in summary, most of the empirical criteria, such as high SST, moist conditions and low vertical wind shear stand out suggesting consistency with the number of TC in ECHAM5. We thus suggest, in agreement with previous observational (Landsea, 2000) and modeling (Wu and Lau, 1992) studies, that it is the geographical distribution of SST anomalies and the corresponding 


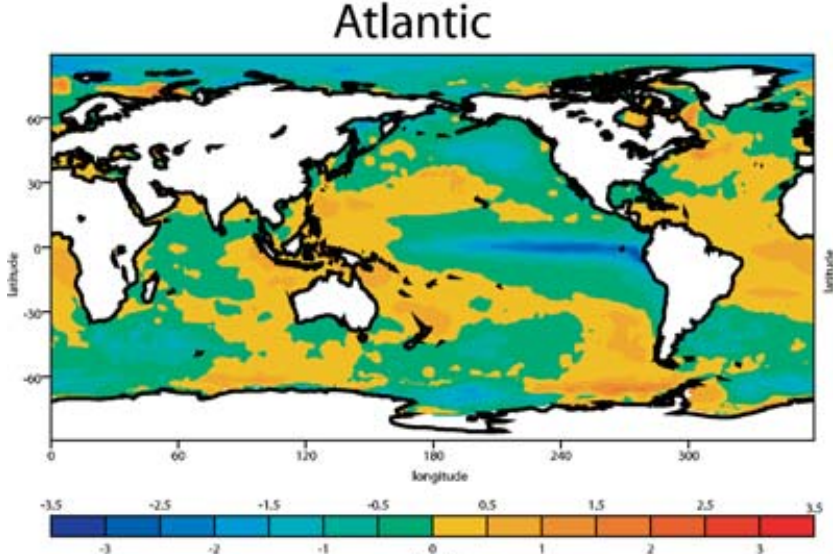

(a) SST
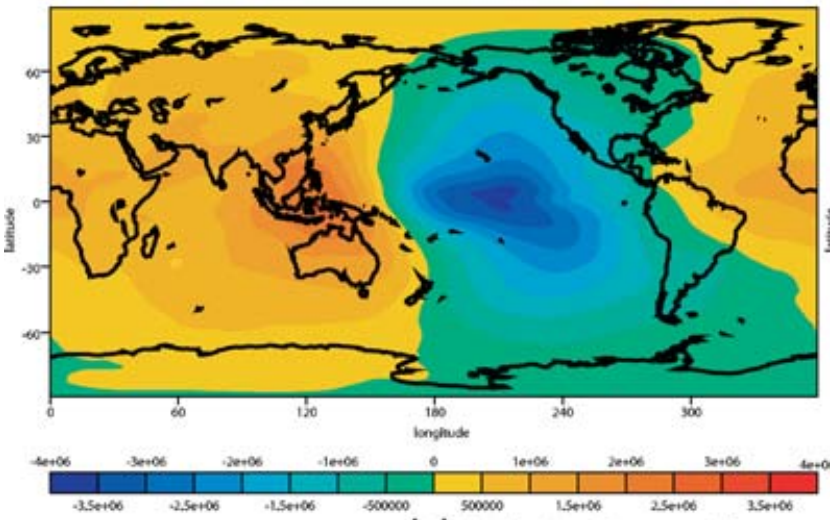

(c) Velocity Potential
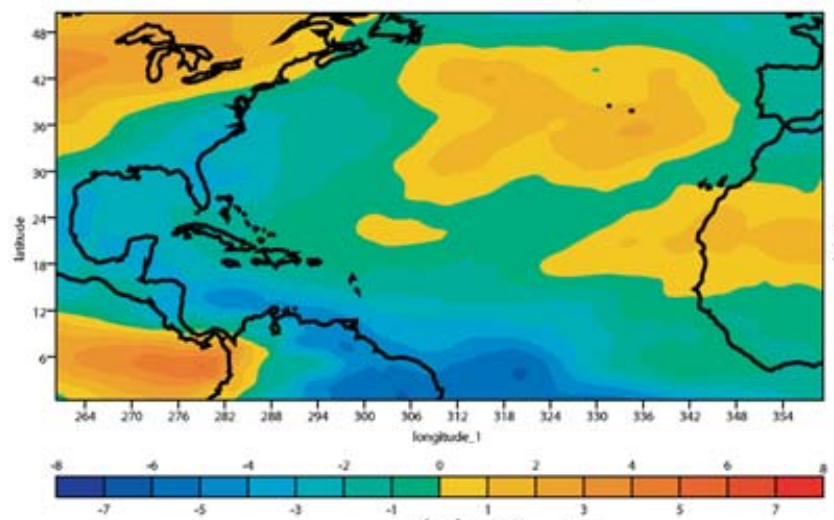

(e) Wind Shear



(b) SST
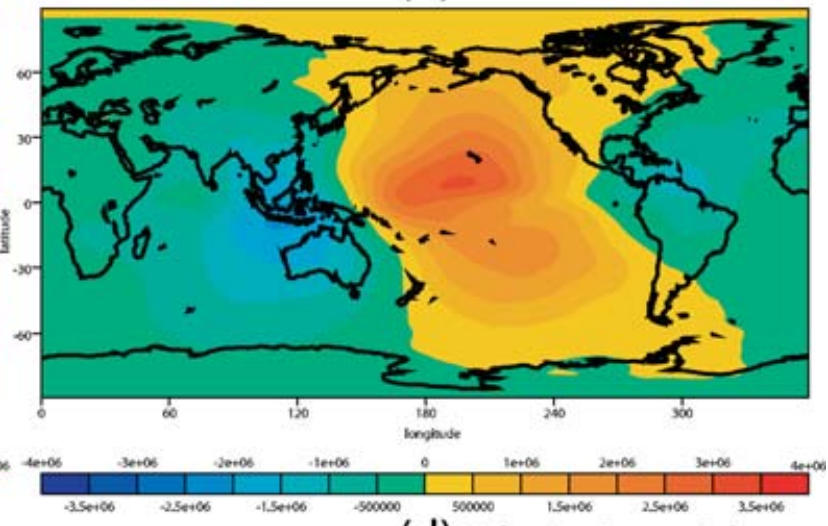

(d) Velocity Potential
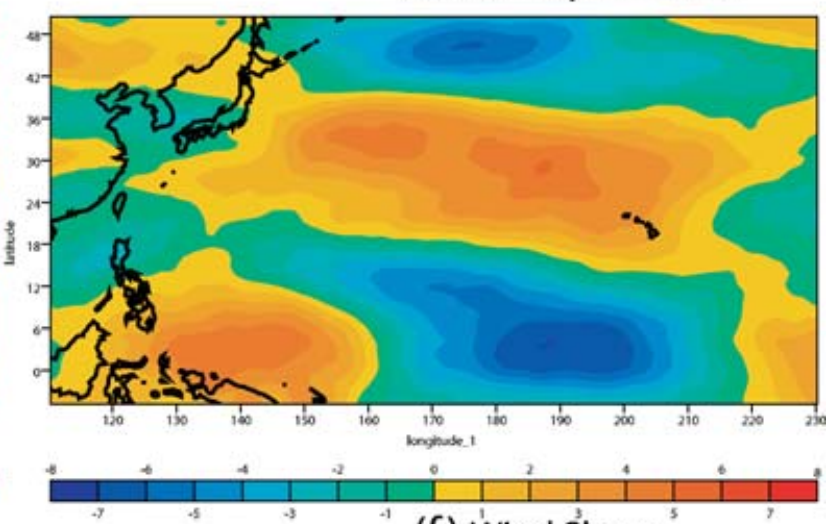

(f) Wind Shear

Fig. 9. Difference between high and low activity years for the Atlantic and Pacific of SST (units of degree Kelvin), 850 hPa velocity potential (units of $\mathrm{m}^{2} \mathrm{~s}^{-1}$ ) and vertical wind shear between 250 and $850 \mathrm{hPa}$ (units of $\mathrm{ms}^{-1}$ ). High years in the Atlantic are 1988 and 1996 and the low years are 1991 and 1997. For the Western Pacific, high years are 1986 and 1994 and the low years are 1988 and 1998. (a) SST for the Atlantic, high-low, (b) SST for the Western Pacific, high-low, (c) velocity potential for the Atlantic, high — low, (d) velocity potential for the Western Pacific, high-low, (e) vertical wind shear for the Atlantic, high-low, (f) vertical wind shear for the Western Pacific, high-low.

response of the large- scale vertical circulation pattern that seems to be crucial for the development of TC in specific regions.

Similar results for ERA40 are shown in Figure 10, based on the same years, and are in both cases very similar to the results for ECHAM5, but the amplitude of the velocity potential pattern is slightly stronger in the model. The wind shear is also mostly in agreement. Other studies (e.g. Oldenbourgh, 2005) have indicated that ECHAM5 has a tendency to over predict the response to El Niño with the stronger velocity potential in ECHAM5 most likely to be a systematic error. 
Atlantic

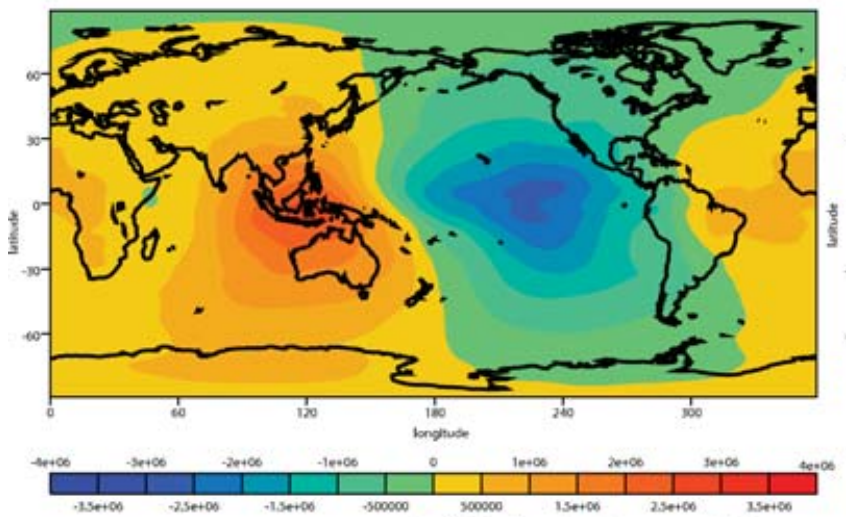

(a) Velocity Potential

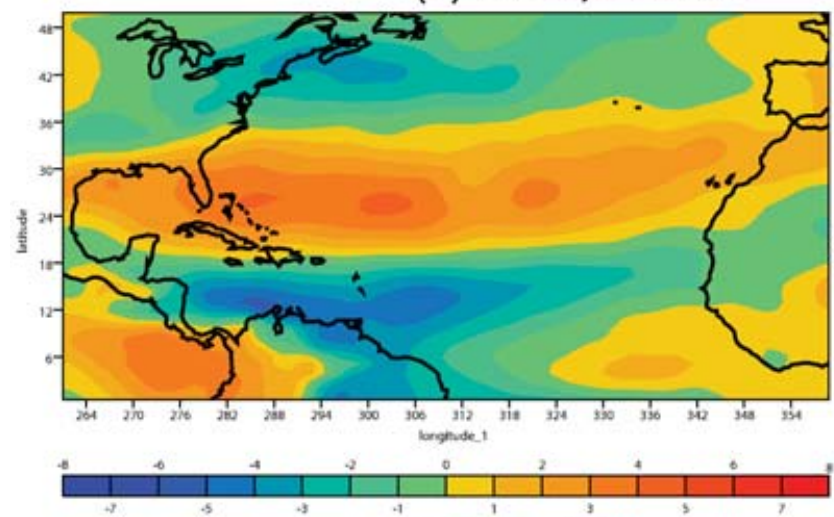

(c) Wind Shear
Western Pacific
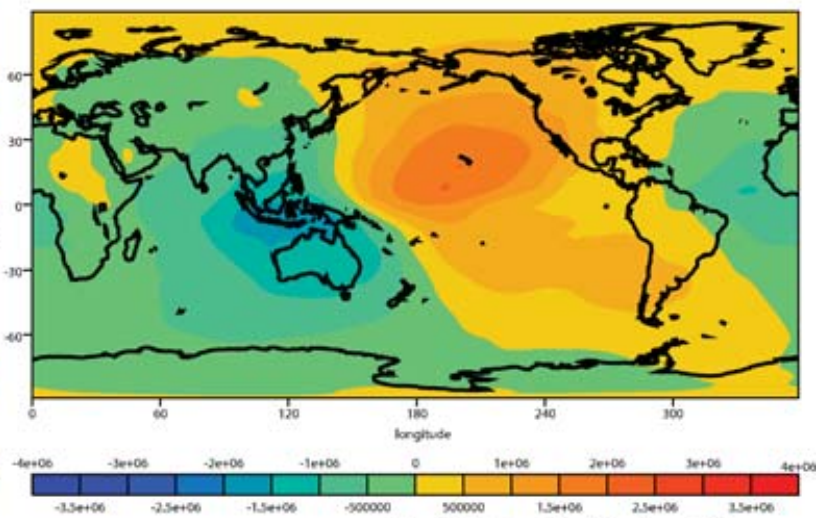

(b) Velocity Potential

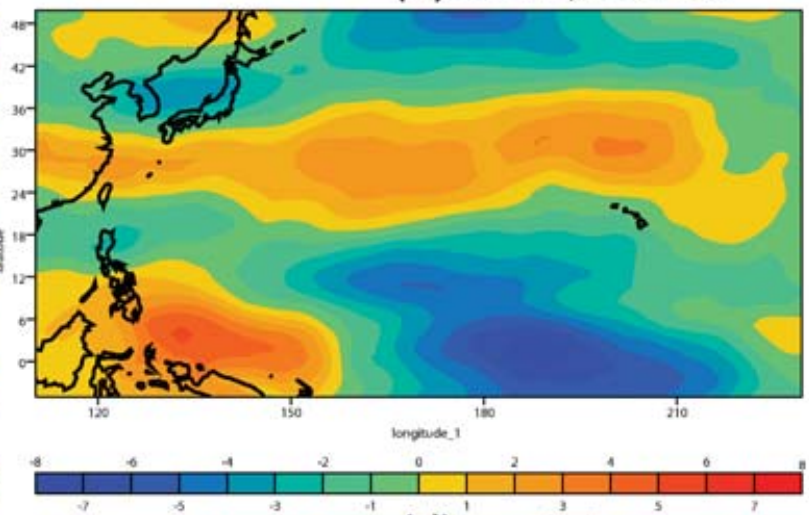

(d) Wind Shear

Fig. 10. Same as Figure 9 but for ERA40 and without SST, (a) velocity potential for the Atlantic, high- low, (b) velocity potential for the Western Pacific, high-low, (c) vertical wind shear for the Atlantic, high-low, (d) vertical wind shear for the Western Pacific, high- low.

\section{Discussion}

In this paper, we have used a novel methodology to identify tropical cyclones in the ECHAM5 model integrated at T156 resolution and in ERA40. The criteria to select TC are based on the (3-D) structure of tropical vortices where we require that the relative vorticity at $850 \mathrm{hPa}$ reaches at least a value of $6 \times 10^{-5} \mathrm{~s}^{-1}$ and at the same time that the difference in vorticity between 850 $\mathrm{hPa}$ and $250 \mathrm{hPa}$ differ by at least the same amount at the T63 resolution and with these criteria fulfilled for at least $1 \mathrm{~d}$. This means that the vortices have a warm core structure, typical of tropical storms. We have determined these conditions by comparing with analyzed tropical storms by the ECMWF operational system. In comparing the ECHAM5 model with ERA40, we have used the same resolution and data for the period 1978-1999 and results are restricted to the Northern Hemisphere. There are several similarities between the model run and ERA40, but the numbers of TC are some $15 \%$ higher in the model run, the lifetime of the generated storms are longer and the intensity is higher. There are considerable similarities in the number of storms in the different regions but the model has more intense storms in the East- ern Pacific and in the North Indian Ocean. There are no storms in ERA40 in the Eastern Pacific reaching category 1 intensity. And there is only one storm in ERA40 reaching an intensity of $50 \mathrm{~ms}^{-1}$ while in the model run there are 74 . In the Northern Indian Ocean the model has too many storms including during the monsoon season. We interpret this as an over prediction of monsoon depressions. The reduced intensity in ERA40 is presumably related to two factors. Firstly, we believe there are an insufficient number of wind observations to support the intensity and secondly, it may be expected that the data-assimilation may have difficulties to properly merge available observations and model information in an intense tropical storm. As will be indicated below the additional wind observations used in JRA25 partly overcomes this problem.

We have also compared model results with observed tropical storms as compiled by the TWC. Such a comparison is difficult as the criteria in selecting storms are not the same. However, when using the maximum sustained wind speed in the boundary layer (in the model we used the wind speed at $925 \mathrm{hPa}$ while TWC use the wind speed at $10 \mathrm{~m}$ ) there are several important differences. Firstly, the model has some $60 \%$ more storms with 
the maximum wind speed higher than $18 \mathrm{~ms}^{-1}$. This could be related to observational difficulties in identifying the maximum wind speed and of course to a deficiency of the model that results in too many weak storms. ERA40 is somewhere in between (Table 2). However, when it comes to intense storms stronger than $50 \mathrm{~ms}^{-1}$ or storms in category 3-5 of the Saffir/Simpson scale, these are significantly underestimated by the model by a factor of 4 . We believe this is related to model deficiencies since it is likely that much higher resolution and possibly an explicit treatment of convection (e.g. Shen, 2006a) will be needed to produce such storms by a model. Nevertheless, in comparing storms that are category 1 or greater $\left(>33 \mathrm{~ms}^{-1}\right)$ the overall distribution is quite realistic. There are interesting differences in the geographical and seasonal distribution, some of them due to insufficient resolution of coastlines and orographic features as in Central America and to over prediction of monsoon depressions as in the North Indian Ocean. There is a general tendency by the model to have proportionally fewer TC in the Atlantic and proportionally too many in the Western Pacific. We suggest that this is not a sampling problem but more likely a systematic feature of the model, which could be related to an overly strong response to the tropical Pacific SST anomalies as noted by Oldenborgh et al. (2005).

The model shows clear interannual variability in a way that shows similarity with ERA40, but with only a minor correlation of $38 \%$. However, in the Atlantic sector and in the Western Pacific the agreement is higher with ERA40. However, the period is too short to determine if the agreement is by chance or whether there is a genuine predictive skill. A series of ensemble integrations with a model at this or higher resolution will be needed. The interannual variability is too strong in the Western Pacific and in the North Indian Ocean where also the number of simulated TC are proportionally too high.

In the Pacific and the Atlantic we have identified a response to Pacific SST, which affects the development of tropical cyclones because of its influence on the large-scale vertical circulation patterns bringing in moist surface air into the genesis regions as well as affecting the vertical wind shear in these areas. This paradigm of large-scale circulation has recently been reiterated by Sugi et al. (2002), and Chauvin et al., (2006) stressing the SST spatial pattern anomaly. We have tested this by studying the difference between two pairs of years representing high and low activity, respectively, in both the Atlantic and in the Western Pacific. This comparison shows that a warm tropical Pacific Ocean as occurs during El Niño events is associated with a marked anomaly in the large-scale divergence circulation leading to ascending vertical winds in the central and Western Pacific and descending vertical winds in the tropical Atlantic. The reverse takes place when there is a cold anomaly in the central tropical Pacific. At the same time the vertical wind shear between 850 and $250 \mathrm{hPa}$ is affected in the regions where tropical storms normally develop. A warm tropical Pacific Ocean also coincides with a stronger than normal vertical wind shear and the opposite in the case of a colder than normal tropical Pacific. We have also shown that the response pattern by ECHAM5 is supported by the analyses from ERA40. We therefore emphasize the importance of considering the distribution of the tropical SST anomaly pattern and the associated atmospheric circulation as being crucial for an understanding of TC, and it would be misleading to focus only on the SST.

It is of utmost importance to clarify to what extent TC might change in a warmer climate. As we are going to address this in a following paper we will only here give some shorter comments. We believe a time period of $21 \mathrm{yr}$ as studied here is too short to be able to identify robust trends as the interannual variations are large. Furthermore, the global observing system is undergoing considerable development which will both affect our ability to observe the intensity of tropical storms and the quality of re-analyses such as ERA40. An increase in the intensity of tropical storms over time in ERA40 can therefore just reflect that there are more reliable observations in the later data.

Several recent studies (Webster et al., 2005, Emanuel, 2005, Sriver and Huber, 2006) have shown that there has been a general increase in intense tropical storms during the last two decades. An interesting quantity used in these studies is the area integrated cube of the wind along the storm track and called the power dissipation (PD) of the storm, (Bister and Emanuel, 1998). As suggested by Emanuel (2005), a simplified power dissipation index (PDI) can be defined as the integrated cube of the maximum wind along the track, $P D I=\int_{0}^{\tau} V_{\max }^{3} d t$, where $\mathrm{V}_{\max }$ is the maximum sustained wind speed at 10 meters. We have calculated the PDI for ERA40 and for ECHAM5 for each individual TC for the portions of the tracks where the conditions $\xi_{\mathrm{I}} \geq 6 \times 10^{-5} \mathrm{~s}^{-1}$ and $\xi_{\mathrm{v}} \geq 6 \times 10^{-5} \mathrm{~s}^{-1}$, at $\mathrm{T} 63$, are satisfied (see section 2), for the $850 \mathrm{hPa}$ vorticity field, i.e. from the first instance to the last. Sriver and Huber (2006) used the best track data produced by TWC and winds from ERA40 integrated over an area around the storm. They found a result in agreement with Emanuel (2005) indicating a huge increase in the 1990s. We have repeated this calculation here, but we have used the TC calculated directly from the ERA40 as well as the maximum wind speed close to the centre of the storm. An identical calculation was undertaken with the ECHAM5. The result from ERA40 shows marked peaks in the years 1992 and 1994 similar to Sriver and Huber. However, ECHAM5 differs with two pronounced peaks in 1981 and 1992. Generally, we would here tend to agree with Chan (2006) that the time period is too short to identify robust trends. Additionally, to calculate the cube of the wind speed may make the result overly sensitive to the maximum wind speed. The fact that the model and ERA40 respond differently suggest a stochastic component, alternatively it may reflect a systematic model bias. Additionally, as has been pointed out by Elsner et al. (2006), the result is highly dominated by high-frequency variability of the PDI and does not strongly indicate a long-term trend or lowfrequency variability. 
A preliminary comparison between ERA40 and the new JRA25 has indicated a number of interesting differences, though consistent with the results shown earlier in this paper for ERA40. JRA25 has a smaller number of storms than ERA40 but a larger number of more intense storms (see Table 2 and Table 3 ) in terms of wind speed. However, JRA25 suffers from the same systematic problem in the Eastern Pacific as ERA40 in terms of a lack of storms with wind speeds greater than $33 \mathrm{~ms}^{-1}$ (not shown). However, a more detailed picture is obtained by comparing those storms that satisfy the $(6,6,4)$ and $(12,6,4)$ criteria in JRA25 with those in ERA40 and ECHAM5 (not shown), this indicates that JRA25 shows a much smaller reduction in numbers proportionally in going from $(6,6,4)$ to $(12,6,4)$ than is seen in ERA40 or ECHAM5. We suggest that this is due to a stronger and more coherent TC structure in JRA25 than in ERA40 in general. That this is so is demonstrated by comparing the same storm in
Figure 2 identified in ERA40 with that identified in JRA25, we have also produced composites (Fig. 12) discussed below. Figure 11a shows the track from JRA25 in more or less the same position as for ERA40 but shortened. The JRA25 version of the storm had a more rapid intensification from an earlier stage in its lifecycle (Fig. 11b), based on vorticity, than in ERA40 and shows a much smoother intensity curve. This is further highlighted by the vertical structure (Fig.11c), which shows a strong warm core structure from earlier in its lifecycle than in ERA40 and is in better agreement with the observed Super Typhoon 21. Finally, these results show consistency with both the central pressure and $925 \mathrm{hPa}$ winds (Fig. 11d), and that although the JRA25 version of the storm has a stronger warm core and stronger winds over the majority of the lifecycle, the actual peak winds and minimum pressure are not that different from ERA40. These differences could have a significant impact on the PDI measure

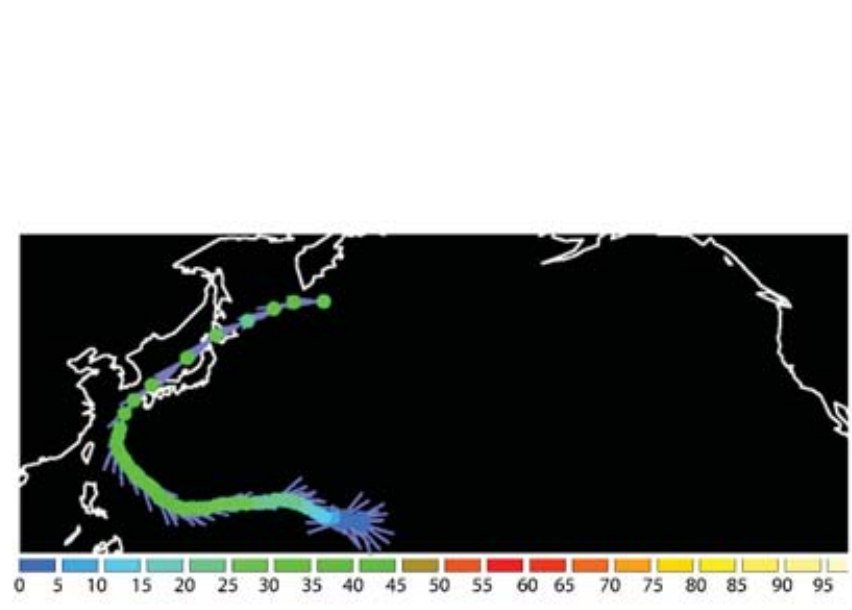

(a)

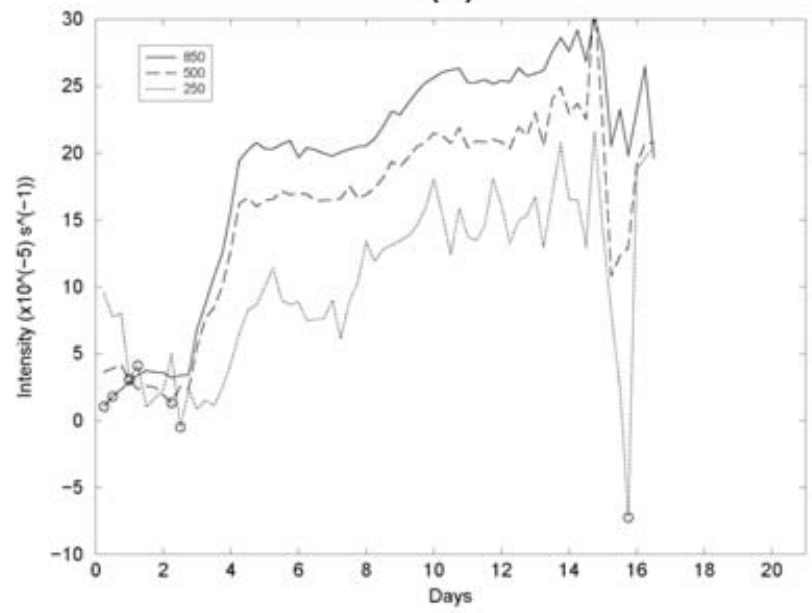

(c)

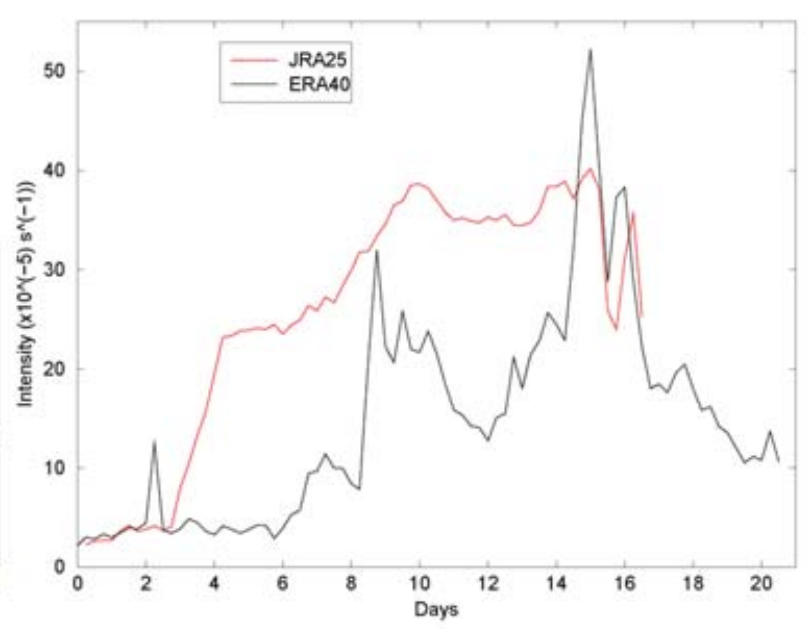

(b)

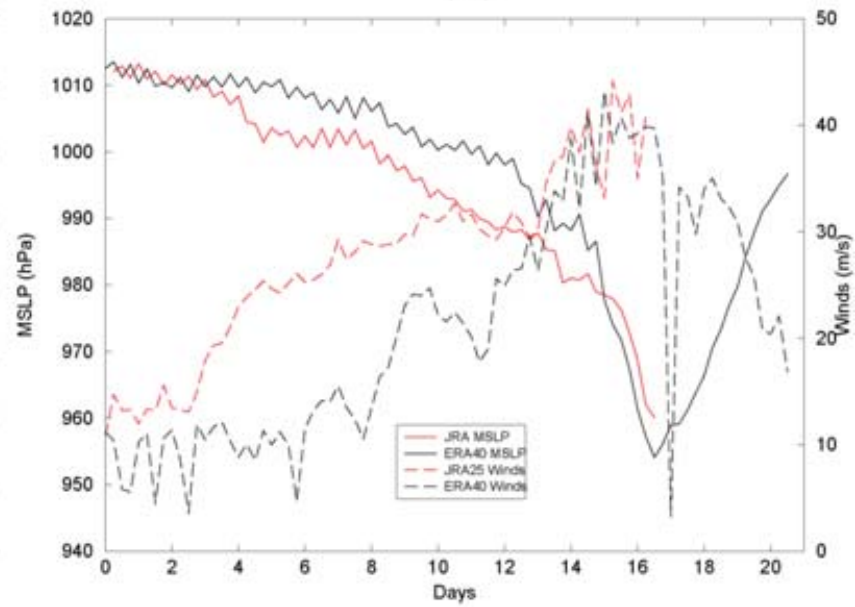

(d)

Fig. 11. (a) Super Typhoon 21 in JRA25, first identified 1991/09/12:18, (b) comparison at full resolution between ERA40 and JRA25 of the intensity for the same storm in the $850 \mathrm{hPa}$ relative vorticity, (c) vertical structure of the same storm in JRA25 as in Figure 2c, (d) comparison at full resolution between ERA40 and JRA25 of the central sea level pressure and maximum wind speeds at $925 \mathrm{hPa}$. 
for example. It was alluded to in section 4 that the differences seen between ECHAM5, ERA40 and now JRA25, both for the examples shown, and the statistics was due to a stronger more coherent warm core structure in ECHAM5, and also JRA25, than in ERA40. To explore this further, we have constructed storm composites from the $10 \%$ of the most intense storms (in vorticity) for both ECHAM5, ERA40 and JRA25, taking the time at which the storms attain their maximum intensity for the averaging. This is done using the T63 vorticity fields. Full details of the compositing procedure will appear in the forthcoming paper (Bengtsson et al., 2007). The storm composites are shown in Figure 12. For ECHAM5 (Fig.12a), there is a strong vertical gradient in vorticity, in particular above $500 \mathrm{hPa}$ suggesting a strong warm core. The radial extent of the composite storm is $\sim 4^{\circ}(\sim 400 \mathrm{~km})$. The low-level intensity is $\sim 30 \times 10^{-5} \mathrm{~s}^{-1}$. For ERA40 (Fig. 12b), there is a much weaker vertical vorticity gradient indicating a weaker warm core structure, the radial extent of the composite storm is also larger than in ECHAM5 and the low level intensity is also weaker. For JRA25, the composite is more similar to ECHAM5 than ERA40 indicating that JRA25 also has a stronger warm core than ERA40 with a similar radial size also similar to that of ECHAM5. The low-level intensity of the JRA25 composite is not as large as ECHAM5 but is larger than ERA40. These results are consistent with all our previous results and discussion.

We suggest that the main reason for the differences between ERA40 and JRA25 is the use in JRA25 of additional wind vector retrievals from historical best track data which are assimilated as though they where from dropsondes (Onogi et al., 2005), in particular those of Fiorino (2002), which were not used in ERA40. Hatsushika et al. (2006) have demonstrated the benefits of the assimilation of the additional observations to TC, including improved detection in the Eastern Pacific and better track position. However, one downside to this is highlighted by the shortened track shown in Figure 11a, which misses the decay stage of the storm. This is possibly due to excessive displacement that violates the tracking constraint for maximum displacement (it could also be due to the formation of a double centre). This can also be seen in the region of Sea of Japan where the JRA25 storm shows a very large jump not seen in either ERA40 or the observations. Thus, while the additional wind observations may result in better simulation of the vertical structure of the storms for some individual storms, it may be detrimental to the changes in position of the storm. This can often occur in analyses where there is a change in observational data coverage and is the bane of feature tracking in both operational and re-analyses.

\section{Concluding remarks}

Re-analyses systems and GCM at sufficient resolution are increasingly capable of assimilating and simulating tropical

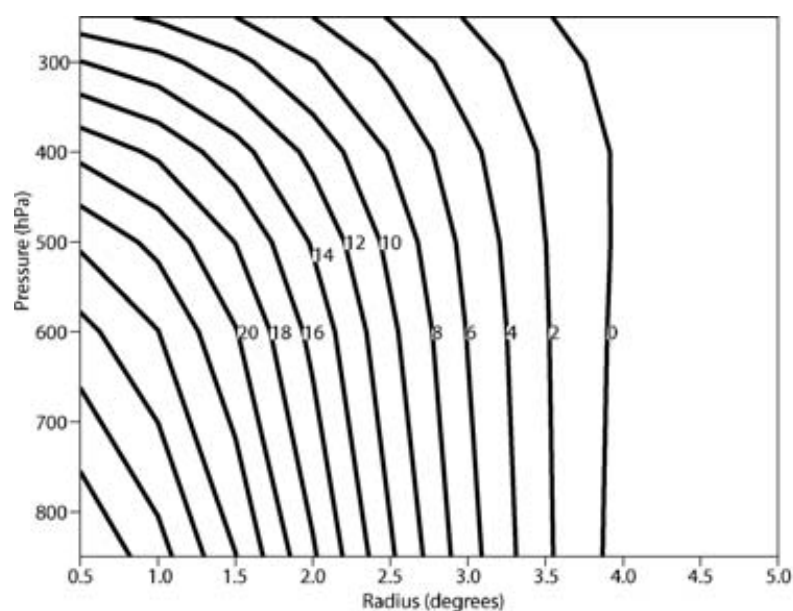

(a) ECHAM5

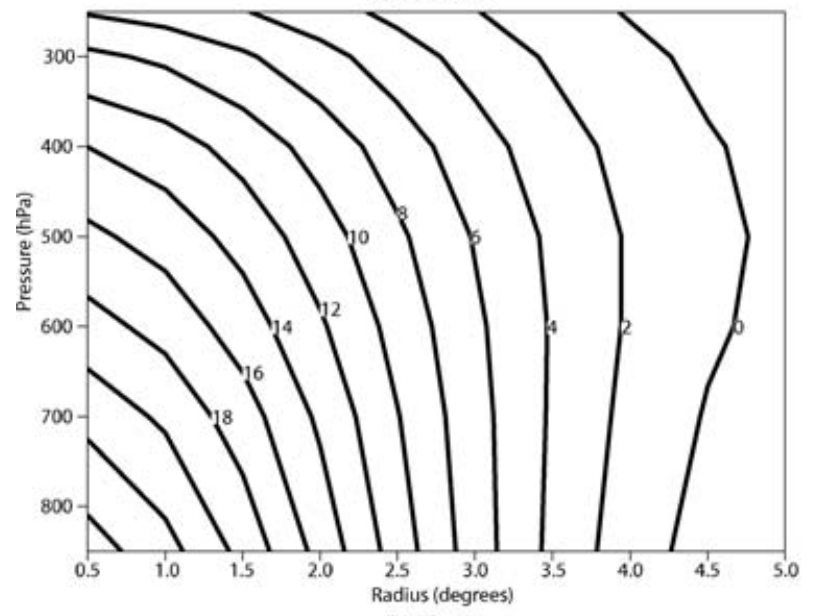

(b) ERA40

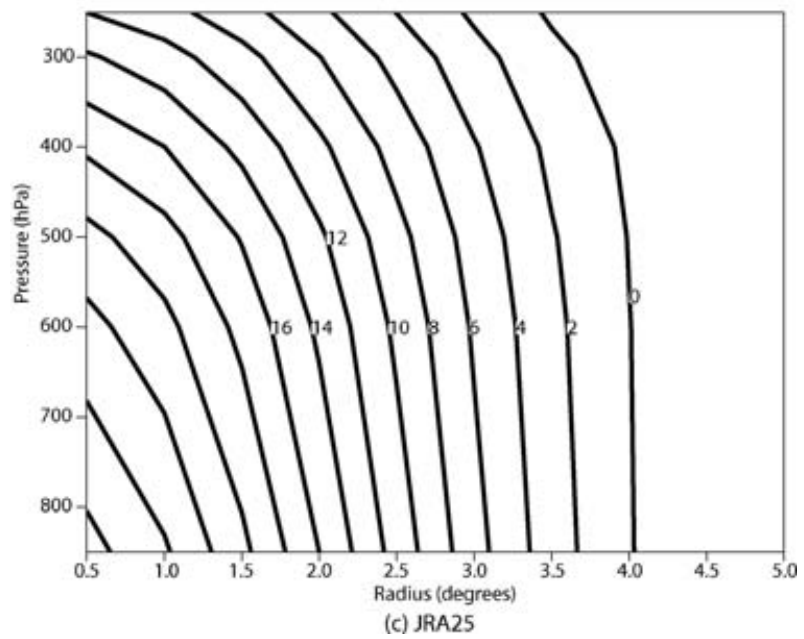

Fig. 12. Composites of vorticity centered on the $10 \%$ of the most intense storms at multiple levels when the storms reach their maximum intensity, (a) ECHAM5, (b) ERA40, (c) JRA25. Units are $10^{-5} \mathrm{~s}^{-1}$. Composites are produced in a cylindrical coordinate system and averaged over the angular direction to produce the radial plots. 
cyclones. The ECHAM5 model at T159 resolution as investigated here also have a realistic interannual variability markedly influenced by the large-scale divergent circulation. To investigate such vortices, we have here used a novel and powerful methodology to calculate the full life cycle of the TC, including their prestorm phase as well as their transformation into extra-tropical cyclones. The main limitation of both the model and the data assimilation systems is an underestimation of the most intense tropical storms. Nevertheless, we consider a diagnostic study of the kind used here as an important tool to determine to what extent high resolution GCM are useful in studying tropical vortices including their variability in time and space, and presumably also how they may change in a warmer climate. A second study to explore this is underway.

In the longer term, we believe the avenue to follow to improve the simulation of the intensity of TC is by firstly increasing the resolution of the GCM as undertaken by Oouchi et al. (2006) and secondly by exploring the impact of alternative ways to treat deep convection including the use of non-hydrostatic modelling.

Future work will explore even higher resolution integrations, and we await the ECMWF interim re-analyses which will use 4-D Var at T255 to compare with. Also, we will use the ability to follow the storms into high latitudes to study the extra-tropical transition of tropical storms.

\section{Acknowledgments}

The authors would like to thank ECMWF for making the ERA40 and operational analyses available via a member state special project and the JMA and Central Research Institute of Electric Power Industry (CRIEPI) for making the JRA25 data available. Also thanks to Dr Ian Bland for making his condor pool available to us for the computation. The ECHAM5 integration was financed in part by the German Ministry for Education and Research (BMBF) under the DEKLIM project and performed on the NEC SX-6 supercomputer installed at the German Climate Computing Centre (DKRZ) in Hamburg. We would also like to thank the anonymous reviewers for their suggestions in improving the manuscript.

\section{References}

Bender, M. A. and Ginis, I. 2000. Real case simulations of hurricaneocean interaction using a high resolution coupled model: Effects on hurricane intensity. Mon. Wea. Rev. 126, 917-946.

Bengtsson, L., Botzet, M. and Esch, M. 1995. Hurricane-type vortices in a general circulation model. Tellus 47A, 175-196.

Bengtsson, L., Botzet, M. and Esch, M. 1996. Will greenhouse gasinduced warming over the next $50 \mathrm{yr}$ lead to higher frequency and greater intensity of hurricanes?. Tellus $\mathbf{4 8 A}$, 57-73.

Bengtsson, L., Botzet, M. and Esch, M. 1997. Numerical simulations of intense tropical storms. In: Hurricanes (eds H. F. Diaz). R. S. Pulwarty Springer-Verlag, Berlin 1997, 67-90.
Bengtsson, L., Hodges, K. I. and Roeckner, E. 2006. Storm tracks and climate change. J. Clim. V19, 3518-3543.

Bengtsson, L., Hodges, K. I., Esch, M., Keenlyside, N., Komblush, L., Luo, J. J., and Yamagata, T. 2007. How many tropical cyclones change in a warmer climate. Tellus, in press.

Bister, M. and Emanuel, K. 1998. Dissipative heating and hurricane intensity. Meteorol. Atmos. Phys. 50, 233-240.

Broccoli, A. J. and Manabe, S. 1990. Can existing climate models be used to study anthropogenic changes in tropical cyclone climate? Geophys. Res. Lett. 17, 1917-1920.

Camargo, S. J. and Zebiak, S. E. 2002. Improving the detection and tracking of tropical cyclones in atmospheric general circulation models. Wea. Forecast. 17, 1152-1162.

Chan, J. C. L. 2006. Comment on 'Changes in Tropical Cyclone Number, Duration and Intensity in a Warming Environment'. Science 311, 1713.

Chauvin, F., Royer, J.-F. and Déque, M. 2006. Response of hurricanetype vortices to global warming as simulated by ARPEGE-Climat at high resolution. Clim. Dyn. 27, 377-399.

DeMaria, M., Mainelli, M., Shay, L. K., Knaff, J. A. and Kaplan, J. 2005. Further improvement to the |statistical hurricane intensity prediction scheme (SHIPS). Weather Forecas. 20, 531-543.

Elsner, J. B., Tsonis, A. A. and Jagger, T. J. 2006. High-frequency variability in hurricane power dissipation and its relationship to global temperature. Bull. Am. Meteorol. Soc. 87, 763-768.

Emanuel, K. 2005. Increasing destructiveness of tropical cyclones over the past 30 yr. Nature 436, 686-688.

Fiorino, M. 2002. Analysis and forecasts of tropical cyclones in the ECMWF 40-yr re-analysis, extended abstract for paper presented at $25^{\text {th }}$ Conference on Hurricanes and Tropical Meteorology, Am. Meteorol. Soc., San Diego, Calif. 29 April-3 May.

Haarsma, R. J., Mitchell, J. F. B. and Senior, C. A. 1993. Tropical disturbances in a GCM. Clim. Dyn. 8, 247-257.

Hatsushika, H., Tsutsui, J., Fiorino, M. and Onogi, K. 2006. Impact of wind retrievals on the analysis of tropical cyclones in the JRA-25 Re-analysis. J. Meteorol. Soc. Japan 84, 891-905.

Henderson-Sellers, A., Zhang, H., Berz, G., Emanuel, K., Gray, W., and co-authors. 1998. Tropical cyclones and global climate change: a postIPCC assessment. Bull. Am. Meteorol. Soc. 79, 19-38.

Hodges, K. I. 1996. Spherical nonparameteric estimators applied to the UGAMP model integration for AMIP. Mon. Weather Rev. V124, 2914-2932.

Hodges, K. I., Hoskins, B. J., Boyle, J. and Thorncroft, C. 2003. A comparison of recent re-analysis datasets using objective feature tracking: storm tracks and tropical easterly waves. Mon. Weather Rev. V131, 2012-2037.

Klotzbach, P. J. 2006. Trends in global tropical cyclone activity over the past twenty years (1986-2005), 2006. Geophys. Res. Lett. 33, doi:10.1029/2006GL025881.

Krishnamurti, T. N., Pattnaik, S., Stefanova, L., Vijaya Kumar, T. S. V., Mackey, B. P., and co-authors. 2005. The Hurricane Intensity Issue. Mon. Wea. Rev. 133, 1886-1912.

Knutson, T. K. and Tuleya, R. E. 2004. Impact of $\mathrm{CO}_{2}$-induced warming on simulated hurricane intensity and precipitation: Sensitivity to the choice of climate model and convective parameterization. J. Clim. 17, 3477-3495.

Landsea, C. W. 2000. El Niño/Southern oscillation and the seasonal predictability of Tropical Cyclones. In: El Niño and the Southern 
Oscillation: Multiscale Variability and Global and Regional Impacts (eds H. F. Diaz and V. Markgraf). Cambridge University Press, 149181.

Nakicenovic, N., and co-authors. 2000. Special Report on Emissions Scenarios. Cambridge University Press, 599 pp.

Ohfuchi, W., Nakamura, H., Yoshioka, M., Enomoto, T., Takaya, K., and co-authors. 2004. 10-km mesh meso-scale resolving global simulations of the atmosphere on the Earth Simulator - Preliminary outcomes of AFES (AGCM for the Earth Simulator). J. Earth Simulator 1, 8-34.

van Oldenborgh, G. J., Philip, S. and Collins, M. 2005. El Niño in a changing climate: A multimodel study. Ocean Sci. Discuss. 2, 267298.

Onogi, K., Koide, H., Sakamoto, M., Kobayashi, S., Tsutsui, J., and co-authors. 2005. JRA-25: Japanese 25-yr re-anlysis projectprogress and status. Meteorol, Q. J. R.. Soc. 131, 3259-3268.

Oouchi, K., Yoshimura, J., Yoshimura, H., Mizuta, R., and co-authors. 2006. Tropical cyclone climatology in a global-warming climate as simulated in a $20 \mathrm{~km}$-mesh global atmospheric model: frequency and wind intensity analysis. J. Meteorol. Soc. Japan 84, 259-276.

Roeckner, E., Bäuml, G., Bonaventura, L., Brokopf, R., Esch, M., and co-authors. 2003. The atmospheric general circulation model ECHAM 5. PART I: Model description MPI-Report 349, $127 \mathrm{pp}$.

Roeckner, E., Brokopf, R., Esch, M., Giorgetta, M., Hagemann, S., Kornblueh, L., and co-authors. 2006. Sensitivity of simulated climate to horizontal and vertical resolution in the echam5 atmospheric model. J. Clim. 19, 3771-3791.

Shen, B.-W., Atlas, R., Chern, J.-D., Reale, O., Lin, S.-J., and co-authors. 2006a. The 0.125 degree finite-volume general cir- culation model on the NASA Columbia supercomputer: Preliminary simulations of mesoscale vortices. Geophys. Res. Lett. 33, doi:10.1029/2005GL024594.

Shen, B.-W., Atlas, R., Reale, O., Lin, S.-J., Chern, J.-D., and co-authors. 2006b. Hurricane forecasts with a global mesoscale-resolving model: Preliminary results with Hurricane Katrina (2005). Geophys. Res. Lett. 33, doi:10.1029/2006GL026143.

Sriver, R. and Huber, M. 2006. Low- frequency variability in globally integrated tropical cyclone power dissipation. Geo. Phys. Res. Letters 33, 11705-11710, doi:10.1029/2006GL026167.

Sugi, M., Noda, A. and Sato, N. 2002. Influence of the global warming on tropical cyclone climatology: An experiment with the JMA global model. J. Meteorol. Soc. Japan 80, 249-272.

Thorncroft, C. and Hodges, K. 2001. African easterly wave variability and its relationship to atlantic tropical cyclone activity. J. Clim. V14, 1166-1179.

Uppala, S. M., Kållberg, P. W., Simmons, A. J., Andrae, U., Bechtold, V. Da Costa, and co-authors. The ERA40 re-analysis. Q. J. R. Meteorol. Soc. 131, 2961-3012.

Webster, P. J., Holland, G. J., Curry, J. A. and Chang, H. R. 2005. Changes in tropical cyclone number, duration and intensity in a warming environment. Science 309, 1844-1846.

WGNE, 1996. AMIP II guidelines. Atmospheric Model Intercomparison Project Newsletter, No. 8, AMIP Project Office, Livermore, CA, 24 pp. [Available from AMIP Project Office, PCMDI, L-264, LLNL, P.O. Box 808, Livermore, CA 94550.]

Wu, G. and Lau, N.-C. 1992. A GCM simulation of the relationship between tropical-storm formation and ENSO. Mon. Wea. Rev. 120, 958-977. 